\title{
Review Article Neural Coding for Effective Rehabilitation
}

\author{
Xiaoling Hu, ${ }^{1}$ Yiwen Wang, ${ }^{2}$ Ting Zhao, ${ }^{3}$ and Aysegul Gunduz ${ }^{4}$ \\ ${ }^{1}$ Interdisciplinary Division of Biomedical Engineering, The Hong Kong Polytechnic University, Hung Hom, Kowloon, Hong Kong \\ ${ }^{2}$ Qiushi Academy for Advanced Studies, Zhejiang University, Zhejiang 310027, China \\ ${ }^{3}$ Howard Hughes Medical Institute, Janelia Farm Research Campus, Ashburn, VA 20147, USA \\ ${ }^{4}$ J. Crayton Pruitt Family Department of Biomedical Engineering, University of Florida, Gainesville, FL 32611, USA
}

Correspondence should be addressed to Xiaoling Hu; xiaoling.hu@polyu.edu.hk

Received 11 April 2014; Revised 23 July 2014; Accepted 10 August 2014; Published 2 September 2014

Academic Editor: Tianming Liu

Copyright (C) 2014 Xiaoling Hu et al. This is an open access article distributed under the Creative Commons Attribution License, which permits unrestricted use, distribution, and reproduction in any medium, provided the original work is properly cited.

Successful neurological rehabilitation depends on accurate diagnosis, effective treatment, and quantitative evaluation. Neural coding, a technology for interpretation of functional and structural information of the nervous system, has contributed to the advancements in neuroimaging, brain-machine interface (BMI), and design of training devices for rehabilitation purposes. In this review, we summarized the latest breakthroughs in neuroimaging from microscale to macroscale levels with potential diagnostic applications for rehabilitation. We also reviewed the achievements in electrocorticography (ECoG) coding with both animal models and human beings for BMI design, electromyography (EMG) interpretation for interaction with external robotic systems, and robot-assisted quantitative evaluation on the progress of rehabilitation programs. Future rehabilitation would be more home-based, automatic, and self-served by patients. Further investigations and breakthroughs are mainly needed in aspects of improving the computational efficiency in neuroimaging and multichannel ECoG by selection of localized neuroinformatics, validation of the effectiveness in BMI guided rehabilitation programs, and simplification of the system operation in training devices.

\section{Introduction}

Neurological rehabilitation usually is a long-term process for patients suffering from trauma or disorders of the nervous system. With the growth of the ageing population across the world, the number of patients with degenerative (e.g., Parkinson disease, amyotrophic lateral sclerosis (ALS)) and vascular disorders (e.g., stroke) has increased substantially. In fact, stroke, a cerebrovascular accident, has been identified as the leading cause of adult disability $[1,2]$. Providing longterm and effective rehabilitation service has been a grand challenge in many countries and has created pressure to current medical care systems [3]. Neural coding, a technology for interpretation of functional and structural information of the nervous system, has contributed a lot to the advancements in neurological rehabilitation.

Successful neurological rehabilitation firstly depends on the accurate diagnosis of the underlying pathology, its anatomical foci, and the effects on functional networks and structural connections. The advancements in volumetric neuroimaging technology now allow us to visualize neural networks in detail from macroscale to microscale levels. For instance, functional magnetic resonance imaging (fMRI) is now commonly used clinically for diagnosis on the cerebral network reorganization after stroke, that is, the macroscale imaging, and with the application of highresolution optical microscopy, the structure and the dynamic connection among a group of neurons could be revealed, that is, microscale imaging [4]. Neural imaging provides not only the diagnostic information, but also the mechanism or theoretical support for designing optimal rehabilitation therapy as an evaluation tool.

Effective treatment is the second important component in the rehabilitation. The traditional physical and occupational therapies are mainly conducted by human therapists, who can interact with a patient and support him/her to complete the desired training tasks. However, with the shortage of the rehabilitation professionals and the growing population of the patients, assistive rehabilitation devices (e.g., rehabilitation robots) are in great demand. The design of rehabilitation devices that can interact with the patients is based on the identification of voluntary motor intention of 
a user. Brain-machine interface (BMI), sometimes termed as brain-computer interface (BCI), is a technique that has been explored to decode such voluntary motor intention from brain signals, for example, electroencephalography (EEG) and electrocorticography (ECoG), which are the neural potentials detected from the scalp and the brain, respectively $[5,6]$. ECoG, also known as intracranial EEG (iEEG), refers to neural recordings from the cortical surface through a surgical incision to the skull. The detection of ECoG is invasive with the electrode array directly attached to the brain; however, ECoG signals have much higher spatial and temporal resolution than the scalp EEG. BMIs with ECoG detection have been mainly investigated in animal models, for example, monkeys, for possible interaction with external systems, such as a computer game, or even a prosthetic robot $[7,8]$. The animal BMI studies paved the road to the application of ECoG BMI to human beings for rehabilitation purposes. BMI technique is important in designing rehabilitation devices for severely paralyzed patients, whose limb motions are hardly to be detected. Besides the neural signals detected from the brain, muscular electricity, that is, electromyography (EMG), also has been used to explore the neural instructions to the muscles. In comparison with neural or neuronal signals, EMG has focalized resolution on individual muscles and relatively higher amplitudes detected noninvasively. Therefore, EMG is also a favorable biosignal in controlling rehabilitation devices for patients with residual muscle functions $[9,10]$.

The third important component in neural rehabilitation is the quantitative evaluation during and after physical training. Rehabilitative treatment is a long-term intervention that usually lasts for years, during which the development of diseases or the progress of recovery needs to be monitored for the adaptation of treatment programs. However, most of the assessment tools used clinically are subjective based on the observation of practitioners, such as the Fugl-Meyer Assessment [11] for evaluation of upper limb motor function and the Modified Ashworth Scores for assessing muscular spasticity [12]. Due to the lack of manpower in rehabilitation industry, even the subjective evaluations are sparse in most of clinical services currently. New methods are needed for quantitative and long-term assessment on the rehabilitation progress and the posttraining follow-ups. Taking advantage of the neural coding technique, it is possible that training devices also can act as evaluation systems. In this review, we summarized the latest breakthroughs in neural coding with the potential application for more effective rehabilitation on neural network imaging and neural informatics in the cortical areas of monkey during dynamic limb motions; we also reviewed the achievements in neural coding by electrocorticographic interpretation in human beings for clinical applications and intelligent robotic system designed for interactive rehabilitation.

\section{Volumetric Neural Imaging}

In the past few decades, one of the most exciting achievements in neural coding studies is volumetric functional imaging, which has enabled monitoring brain-wide neural activities at precise locations. In this section, we reviewed the recent advancements of the macroscale fMRI and the microscale/mesoscale optical microscopy, the two major classes of volumetric imaging techniques that have been used or have potential applications for rehabilitation. We also highlighted the application of volumetric imaging on neural network reconstruction, which is expected to have fundamental impact on rehabilitation.

2.1. Macroscale Imaging-Functional MRI. MRI opens a new window for observing the brain noninvasively. By measuring wave energy emitted from hydrogen atoms excited by a magnetic field, MRI can produce 3D images of anatomical structures or physiological status of a brain. In particular, fMRI, which measures the BOLD (blood-oxygen-level dependent) effect related to neural activities, provides a unique opportunity of recording activities of the whole human brain at a relatively high resolution. We can study neural encoding and decoding with fMRI, which presents individual voxels as the basic coding unit.

Encoding of fMRI predicts voxel-wise activities given certain stimuli. Kay et al. developed a visual encoding model with four components, including the set of stimuli, the features of stimuli, ROI in the brain, and the algorithm of model estimation [13]. This strategy can be adapted for encoding motion, where the first two components become the set of movements and the features of movements. Decoding of fMRI signal has been extensively studied. It maps voxel dynamics to external stimuli [14], motor behaviors [15], or even high-level cognitive states [16]. While general linear models have been successfully used to build mapping models, there are significant efforts underway for applying modern machine learning techniques [17], such as kernel methods [18], random forests [19], and manifold learning [20] for the same purpose.

Another exciting development in fMRI decoding is realtime fMRI (rtfMRI) [21], which takes advantage of online processing of fMRI images. Set up with decoding models trained offline, rtfMRI returns decoding results as interpretable feedback to the human subject within a short delay after acquisition. The process is fast enough for the subject to modulate the brain activity in the sense of real time. Powerful parallel processing frameworks, which are becoming more and more affordable nowadays, can further improve the decoding speed and potentially clear any bottlenecks in computational modules [22]. This creates an opportunity for building high degree-of-freedom noninvasive brain-machine interfaces using fMRI.

2.2. Microscale/Mesoscale Imaging-Optical Microscopy. At the microscopic/mesoscale level, the most common functional imaging technique is calcium imaging, which mainly uses small fluorescence dyes (e.g., fura-2), or genetically encoded fluorescence proteins (e.g., GCaMP), to measure the concentration fluctuation of free calcium ions in response to electrical signals. Calcium imaging can be used to measure a large population of neurons in vivo. Assisted by genetic 
TABLE 1: Comparison of functional brain imaging methods.

\begin{tabular}{|c|c|c|c|c|}
\hline & $\begin{array}{l}\text { Temporal } \\
\text { resolution }\end{array}$ & $\begin{array}{l}\text { Spatial } \\
\text { resolution }\end{array}$ & Advantage & Limitation \\
\hline fMRI [144] & $\geq 0.5 \mathrm{~s}$ & $\geq 1 \mathrm{~mm}$ & $\begin{array}{l}\text { Noninvasive whole } \\
\text { brain imaging }\end{array}$ & Indirect measurement \\
\hline $\begin{array}{l}\text { Optical } \\
\text { microscopy [23] }\end{array}$ & $\sim 0.2 \mathrm{~s}$ & $\sim 0.2 \mu \mathrm{m}$ & Single cell resolution & Unsuitable for human subject \\
\hline Scalp EEG [145] & $\sim 1 \mathrm{~ms}$ & $\begin{array}{l}\geq 2 \mathrm{~cm}(128 \\
\text { channels })\end{array}$ & $\begin{array}{l}\text { Noninvasive whole } \\
\text { brain recording }\end{array}$ & $\begin{array}{c}\text { Low spatial resolution; signals are easily } \\
\text { contaminated by noises (e.g., EMG, } \\
\text { motion artifacts, etc.) }\end{array}$ \\
\hline ECoG $[95-99,146]$ & $\sim 5 \mathrm{~ms}$ & $\sim 10 \mathrm{~mm}$ & $\begin{array}{l}\text { Long-term and } \\
\text { continuous recording }\end{array}$ & $\begin{array}{l}\text { Invasive recording by attaching the } \\
\text { electrode array on the surface of the brain }\end{array}$ \\
\hline
\end{tabular}

engineering, it can measure specific types of neurons or neurons at specific locations. Recent breakthroughs demonstrated the real power of calcium imaging for studying the whole brain at the single cell level: Schrödel et al. recorded $\sim 70 \%$ of head neurons of C. elegans using wide-field temporal focusing [23] and Ahrens et al. recorded more than $80 \%$ of all neurons of the larval zebrafish brain using light-sheet microscopy [4].

Studying neural coding with microscope imaging, however, is a relatively new research area, which has few original methods designed for special properties of calcium imaging data. Therefore, this presents a new opportunity and challenge to computer scientists and engineers to develop innovative computational algorithms and tools. One major challenge would be the big data problem, because scanning the whole brain at the microscopic scale will produce terabytes or even petabytes of data. Decomposing the data into tractable components and mapping them onto a lowdimensional feature space are a key to revealing unknown brain dynamics.

Although its direct clinical application is yet to be clear, microscopic imaging can revolutionize rehabilitation by providing the mechanism or theoretical support for designing optimal rehabilitation therapy. This will rely on animal studies, which are more accessible resources for understanding the human brain than the human brain itself. The relatively small scale of animal brains offers practical opportunities for understanding a complete nervous system at the single cell level. Due to the fundamental similarities among the motor systems of all animal species, we can build disease models on animals to study problems associated with rehabilitation. For example, Li et al. has developed a C. elegans model of ALS to evaluate the role of autophagy in the disease [24]. For the vertebrate species, there are mice models for studying motor axon regeneration and muscle reinnervation [25] and zebrafish models for studying brain disorders [26]. Another obvious advantage of setting experiments with animal models is the possibility of using a rich set of genetic tools for targeting specific neurons and manipulating neuron functions [27]. The recent development of optogenetics especially has allowed us to control neural activities more precisely than ever before. Combining optogenetics with calcium imaging will definitely provide a powerful tool for observing neural activities when activating or inhibiting a specific set of neurons [28].

2.3. Neural Network Reconstruction. The human brain is a highly dynamic network generating coordinated activities of billions of connected neurons. In this sense, neural rehabilitation is basically the recovery of impaired neural networks. Therefore, the advancement of rehabilitation techniques relies on how well we understand neural coding in the neural network, which in turn requires reconstructing functional network or connectome from real data.

Electrophysiological recordings have been used to reconstruct functional neural networks at different scales [2931], but they have fundamental limitations in resolution or coverage (Table 1). For example, EEG/ECoG can only provide networks with a low spatial resolution due to their recording sites outside of the brain. On the other hand, while extracellular electrophysiological recordings of neuron activities have the single cell resolution, they are limited to a small subset of neurons with obscure identities.

To reconstruct a functional network with better resolution-scale trade-off, we must take advantage of volumetric functional imaging. A common practice for mapping the functional connectivity of human brains is to compute region correlations of the spontaneous fluctuation of the BOLD effect in resting-state fMRI imaging [32]. Various statistical correlation analysis approaches, such as clustering [33], independent component analysis [34], and Bayesian network [35], have been successfully applied. These approaches should also be applicable to microscopic functional imaging, which is currently at the stage of delivering whole brain data at single cell resolution.

We can also reconstruct neural networks anatomically and then infer the functional connectivity. This can be done by diffusion tensor imaging at the macroscopic scale [36], serial two-photon tomography at the mesoscale [37], and fluorescence microscopy [38] or electronic microscopy [39] at the microscopic scale. Although structural neural networks do not provide functional connections directly, they have rich clues about how information processing is implemented in the brain. For example, structural analysis of a circuit in the Drosophila optical lobe has, for the first time, shown that 


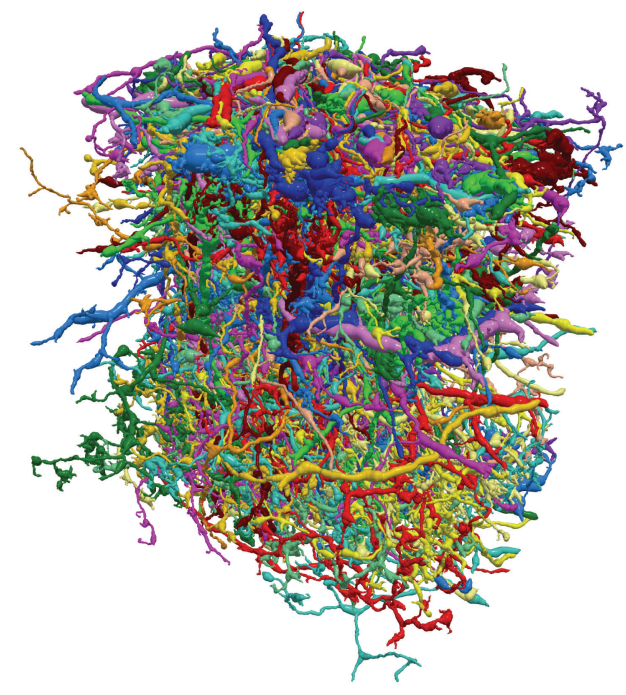

(a)

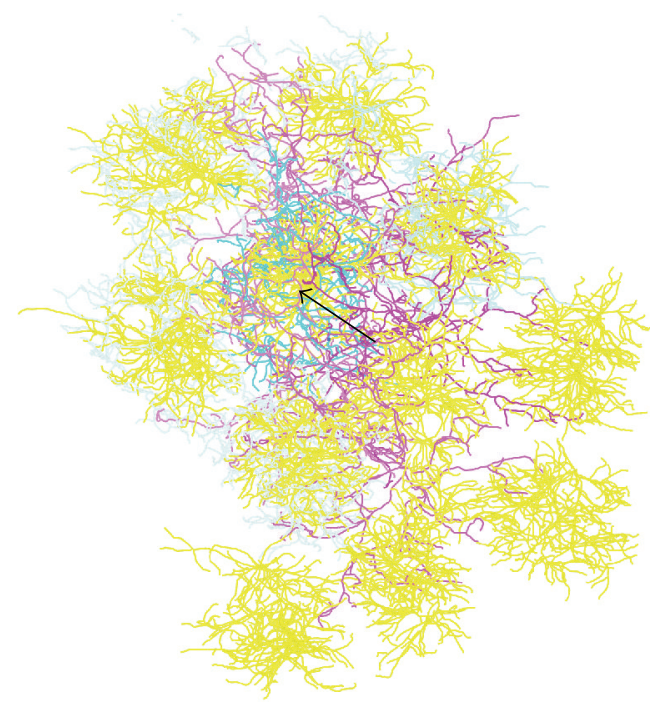

(b)

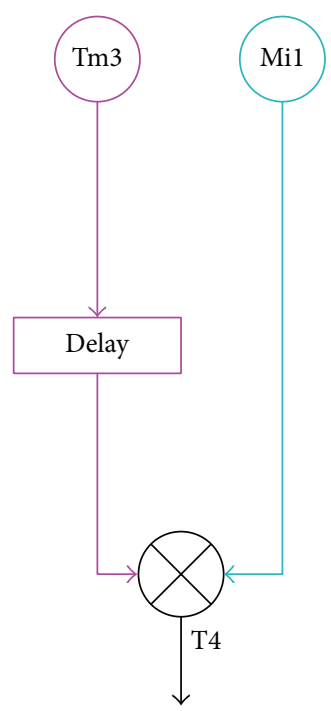

(c)

Figure 1: The motion detection circuit suggested by a connectome reconstructed from the Drosophila brain [39]. (a) Visualization of 379 neurons in the connectome, which is a part of the optical lobe; (b) the offset (black arrow) of the receptive fields computed from the circuit, which involves L1 (yellow), Tm3 (magenta), Mil (cyan), and T4 neurons, suggests a potential implementation of (c) the Hassenstein-Reichardt model.

the brain computes the offsets of receptive fields to detect motion (Figure 1) [39]. How to integrate structural and functional neural networks would be a very interesting research topic, which holds promise for revealing fundamental rules of neural computation.

Undoubtedly, a better understanding of the structural and functional properties of the brain network will lead to more accurate simulations of the brain activities and behavior outputs. Recently, Eliasmith et al. reported a largescale human brain model called "Spaun," which can drive a physically modeled arm to draw pictures by following visual stimuli [40]. It is possible to use a similar framework to simulate motor behaviors given specific neuron degeneration or impaired conditions. These models can be further combined with realistic muscle-based locomotion models, such as those used in computer graphics [41], to assist in diagnosis or treatment planning.

\section{Neural Coding in Brian Machine Interface (BMI)}

Brain-machine interfaces exploit the spatial and temporal structure of neural activity of the brain to directly control a prosthetic device. This emerging field has been mainly inspired by the requirements of restoring interactions between the environment and the individuals with severe sensorimotor deficits through BMI-controlled systems. For example, a tetraplegic patient can feed herself with chocolate using a BMI-controlled robot arm [42].

3.1. BMI with Animal Models. Since the first experimental demonstration using the primary motor cortical signals of a rat to control a lever press [43], nonhuman primates have been utilized as ideal subjects for BMI studies [42-54] due to the similar functional brain structure as human beings [55], which enables the implantation of multiple electrode arrays in different motor cortical regions, and the better capability to perform complicated tasks than other animal models. Starting with a standard center-out movement task in primates, in which monkey's neural activities were found to be tuning to the directional movement [56], the ensemble of the neural firings could be used to predict more complicated arm movement in a $2 \mathrm{D}$ computer cursor control or $3 \mathrm{D}$ reaching and grasping, as well as the gripping force [47, 51, 57-59]. In 2008, Schwartz's group realized a real-time cortical control of a prosthetic robot arm for self-feeding without the real movement of the monkey's arm [53], which is an important step close to the later clinic BMI applications on human $[42,45]$.

In a typical motor BMI framework, neuronal activity (local field potentials, single-unit activity, and multiunit activities) is synchronously collected from microelectrode arrays implanted into multiple cortical areas (primary motor cortex (M1), premotor cortex (PMA), supplementary motor cortex (SMA), primary somatosensory motor cortex (S1), posterior parietal area (PP), etc.) while the subjects are performing movement tasks. Several signal-decoding approaches have been applied to extract the functional relationship between the neural recordings and the subjects' kinematics. The decoder implements a model to predict movements and control a prosthetic robot arm or computer. The first issue in BMI decoding is the choice of the motor parameters, such as position, velocity, acceleration, gripping force, and even EMG signals, which are probably more promising for the patient to accept as the brain-muscular 

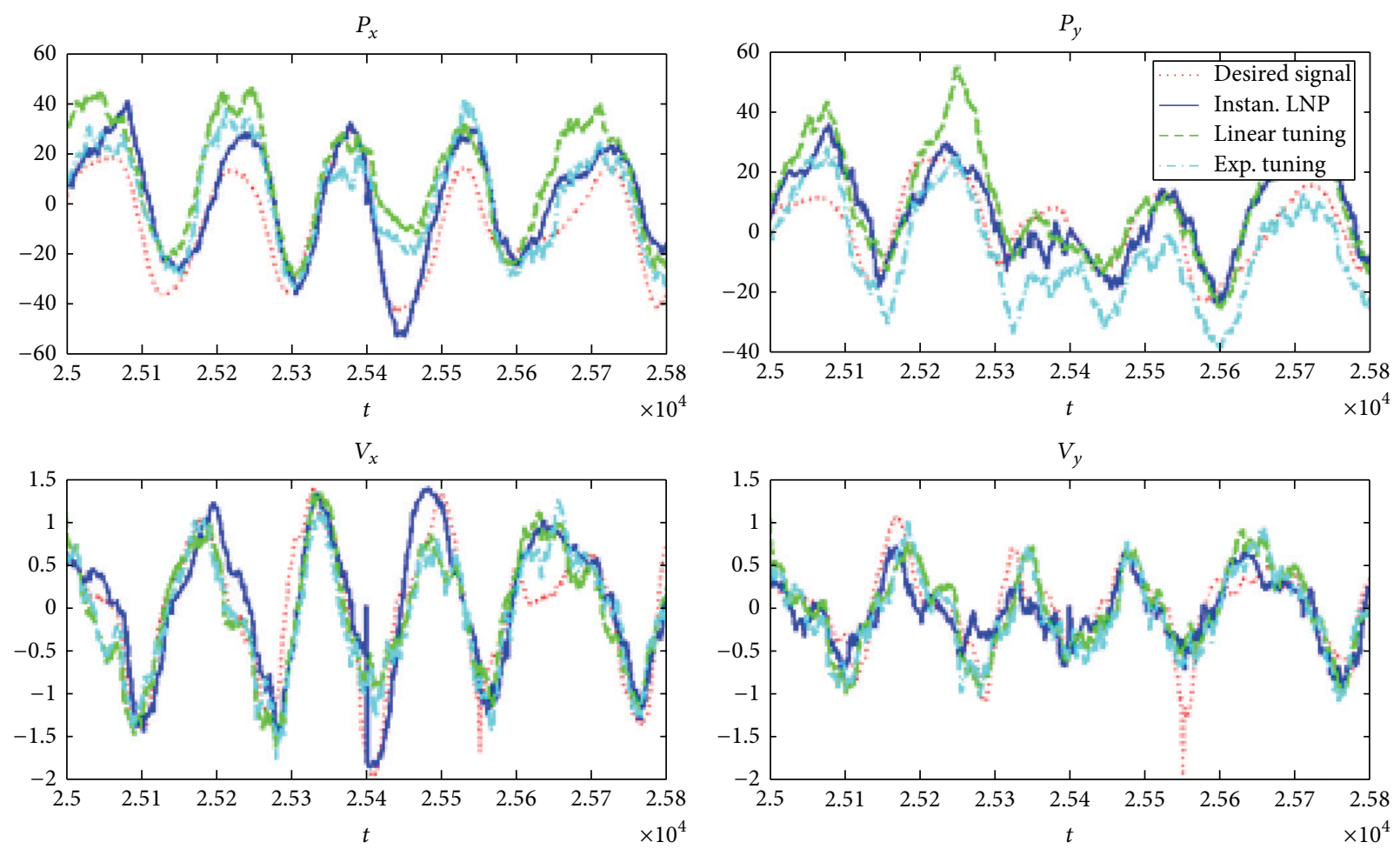

$A_{x}$
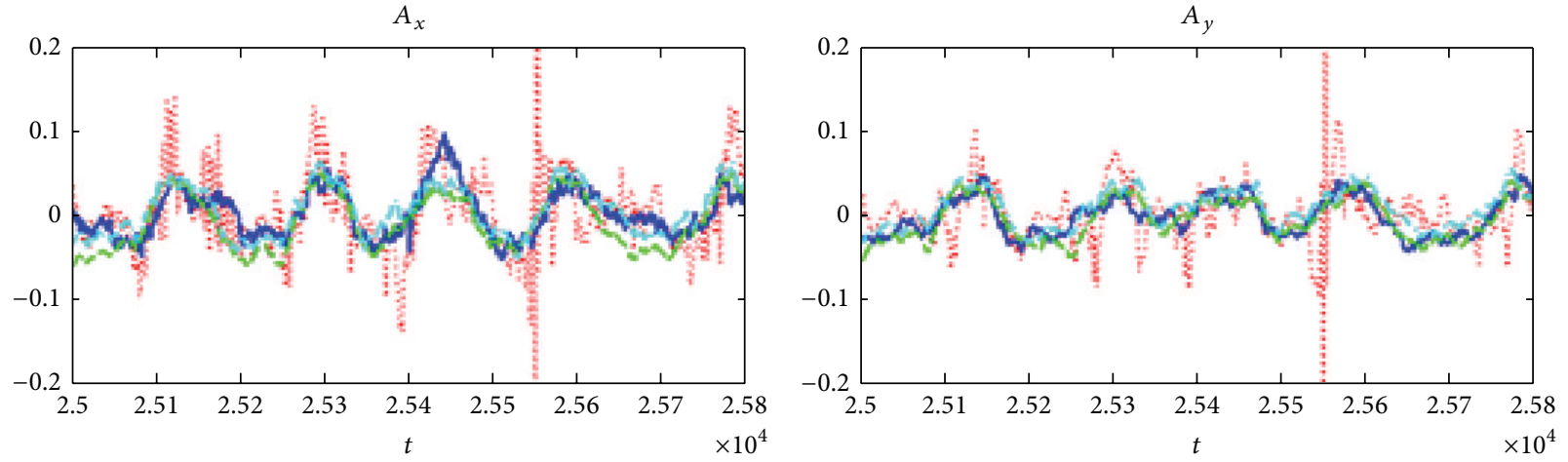

(a)

(b)

FIGURE 2: The reconstructed kinematics for a 2D reaching task by different tuning models from 185 neurons for 1000 testing samples (10 ms for each time instance) [67]. The left and right panels depict the reconstructed kinematics for the $x$-axis and the $y$-axis, respectively. The three rows of plots from top to bottom display the reconstructed position, the velocity, and the acceleration, respectively. In each subplot, the dotted red line indicates the desired signal, the solid blue line indicates the estimation using the proposed instantaneous LNP model, the dashed green line indicates the estimation using linear tuning, and the dot-dashed cyan line indicates the estimation using exponential tuning.

interfaces other than the stiff robot [46]. The second issue is to find a decoding algorithm to translate the cortical activities accurately. Many decoding methodologies use binned spike trains to predict movement based on linear or nonlinear optimal filters $[50,51,54,58,60]$, but lack of further interpretation of the neurological dynamic tuning properties. Another method that derives the movement states probabilistically from the neural tuning model is to use a Bayesian formulation $[61,62]$. It shares the parallel that the brain makes decisions based on prior knowledge [63]. As the binning on the spike does not exploit spike timing structure and may exclude rich neural dynamics in the modeling, the adaptive point process filtering methods have been developed to directly derive the kinematics from the spike trains $[64,65]$ with the modeling of the neural tuning properties to the instantaneous time instance and the connectivity among the neural ensemble $[66,67]$. For example, Wang's work developed a novel, online, and encoding model that uses the instantaneous kinematic variables (position, velocity, and acceleration in $2 \mathrm{D}$ or $3 \mathrm{D}$ space) to estimate the mean value of an inhomogeneous Poisson model [67]. Figure 2 shows an implementation of an instantaneous tuning model in sequential Monte Carlo point process estimation based on spike timing, which provided statistically better kinematic reconstructions than the linear and exponential spike-tuning models in monkey.

Aiming at the computational efficiency for the portable BMI devices, researchers also developed techniques to ascertain the neurons that relate the most to the movement 

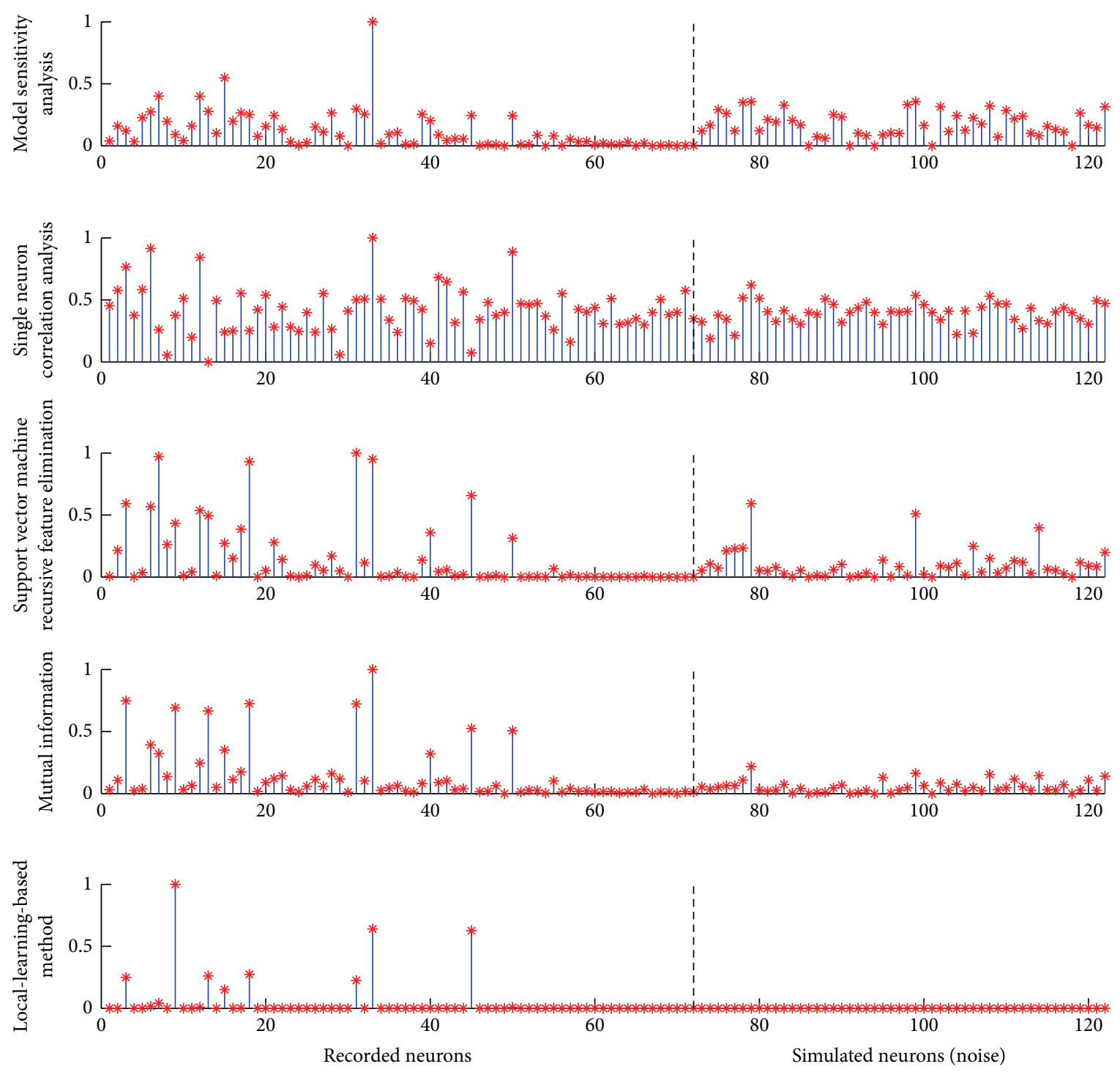

FIGURE 3: Distributions of the neuronal weights calculated by different methods, including single neuron correlation analysis, model sensitivity analysis, support vector machine recursive feature elimination, mutual information, and local-learning-based method. Neurons on the left side of the vertical dash line show real recordings. Neurons on the right are simulated and generated independently from the task. The five approaches show the different abilities of eliminating noisy neurons. The weights of the simulated neurons, learning from our proposed method, are all close to 0 , while many of them are assigned with relatively large values by other methods [70].

task and gain better understanding of the individual neuron firing behavior [58, 68-70]. In Xu's work, a local-learningbased method was proposed to perform neuron selection for the gesture prediction in a monkey's reaching and grasping task [70]. The algorithm effectively ascertained the neuronal importance without assuming any coding model and provides a high performance with different decoding models. The method showed better robustness of identifying the important neurons with noisy signals presented, as shown in Figure 3. The ascertainment of the important neurons helped to inspect neural patterns visually associated with the movement task (Figure 4).

One important issue for clinical BMI application is to incorporate prosthesis devices into body representation and make it feel like the subject's own limb [71]. Introducing the sensory feedback including visual, auditory, and tactile cues, BMI therefore becomes a close loop system [72]. Although visual or auditory information is fed back to the subject in previous BMI designs $[50,51,58]$, researchers investigated the possibility to embed peripheral tactile and proprioceptive signals into the prosthesis operation. O'Doherty et al. [48] implemented intracortical microstimulation techniques directly on the cortical area (S1) of the monkey in a BMI task, in which the monkey could distinguish 3 different targets due to the simulated sense of "touch" in the brain. However, arguments still remain whether the true sense of "touch" is reproduced or the monkey just learns the link between the targets and the electrical "tingling." Other than intracortical 

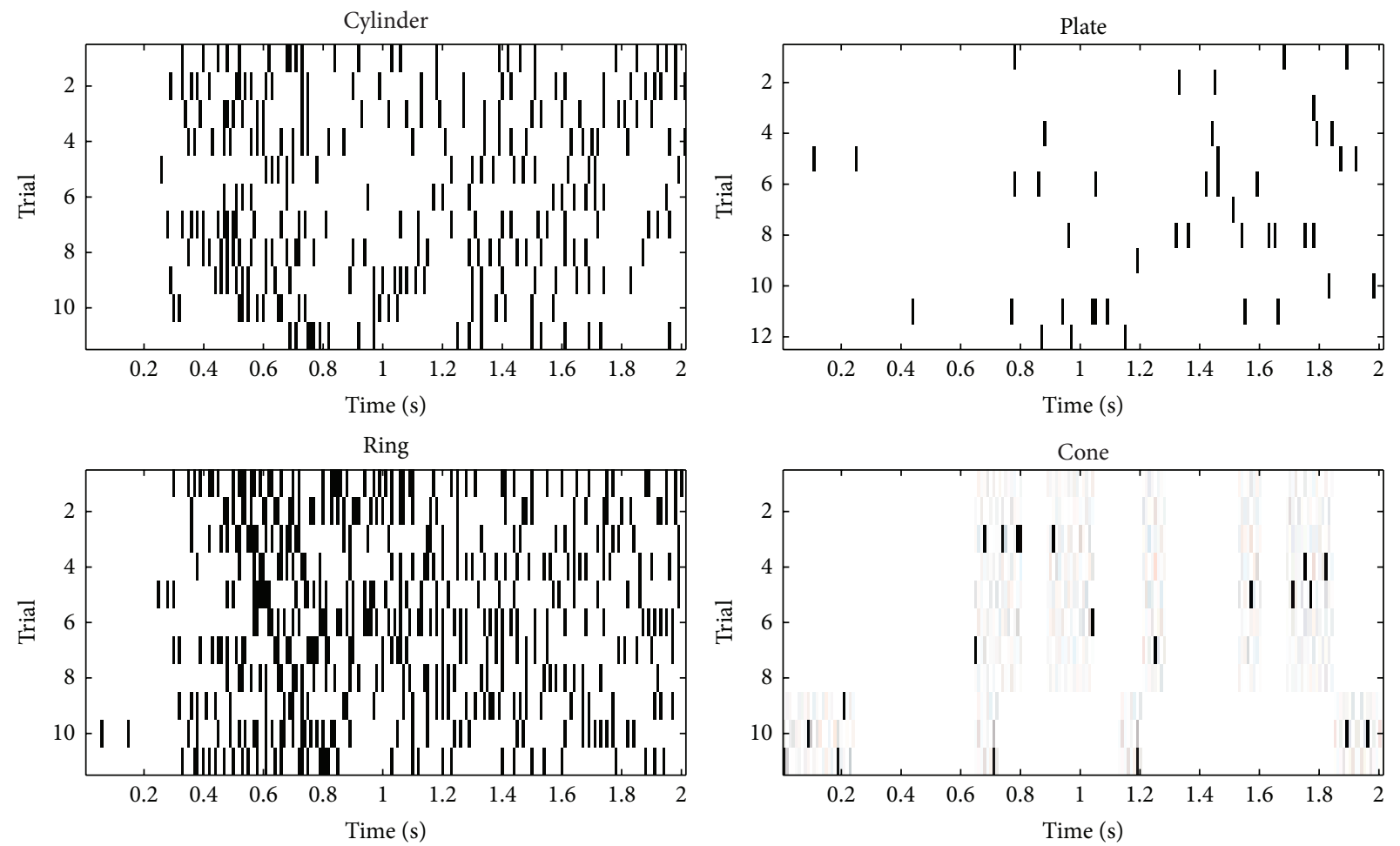

(a)
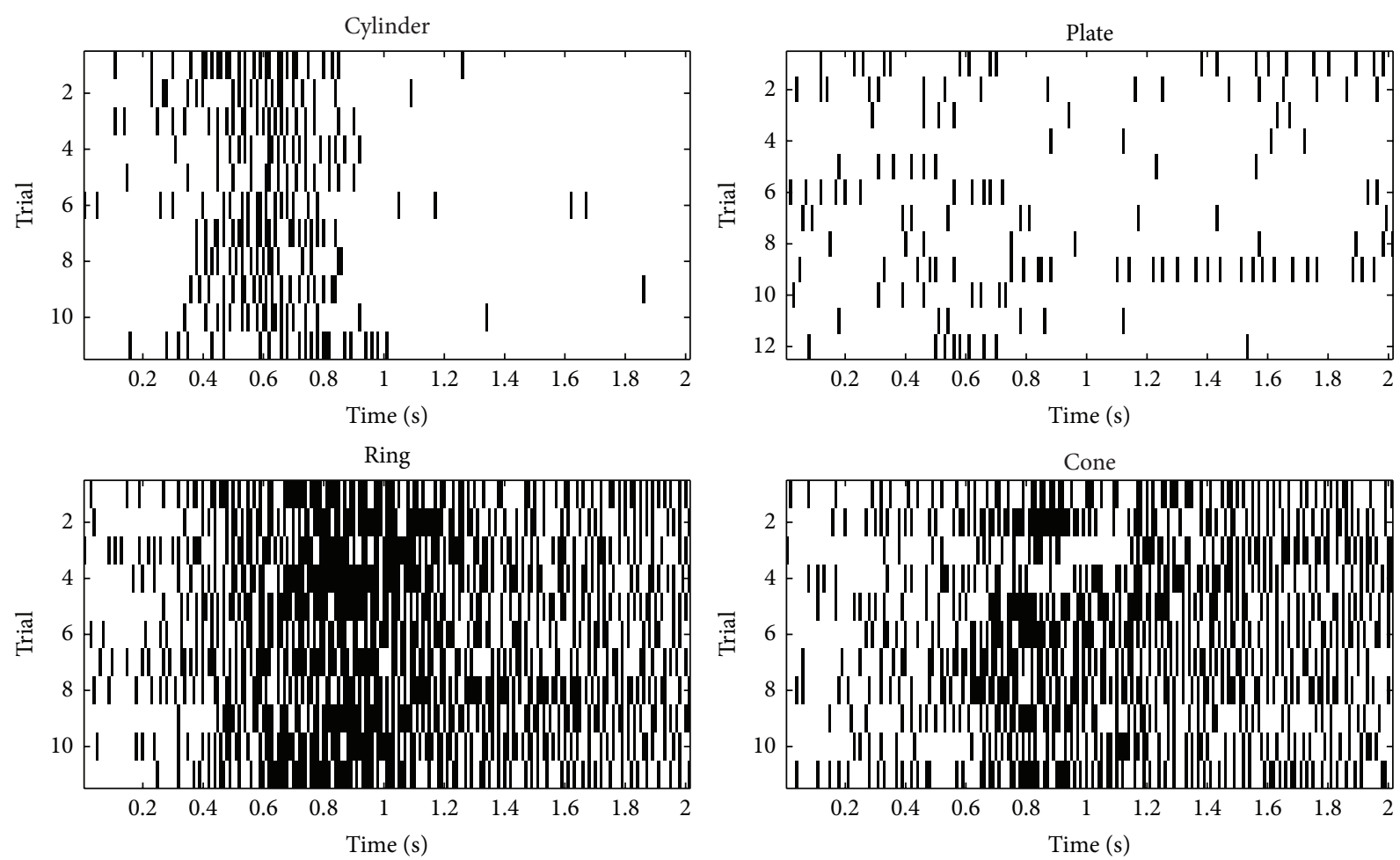

(b)

Figure 4: Temporal activities of the top two neurons corresponding to different grasping targets. The light was on at time 0; the object was grasped around time $1 \mathrm{~s}$ and was held until time $2 \mathrm{~s}$. (a) The first neuron. (b) The second neuron. In each block, the grasping target in the upper left plot is a cylinder. The target in the upper right plot is a plate. The target in the bottom left plot is a ring. The target in the bottom right plot is a cone. Neuron 1 fires more frequently when the target objects were the cylinder and the ring, while much less for the plate and the cone. The activity of neuron 2 clearly distinguished the group of cylinder and plate and the group of ring and cone. Furthermore, neuron 2 ceased to fire around time $1 \mathrm{~s}$, which separated the reaching period and the grasping period [70]. 
microstimulation, optogenetic techniques become promising to active certain types of cells as stimulation to generate the peripheral tactile and proprioceptive feedback due to better temporal and spatial precision, easier manipulability, and less side effects [73]. Although study shows that mice could be guided to run in a circle by such techniques [74], there are few reports on monkey to appear difference in the behavior level using optogenetics as stimulation [75].

In the closed-loop BMI application, the subject needs to learn how to operate a BMI system using biofeedback. The neuroplasticity, induced by biofeedback, could help the subject adjust brain activity to better adapt to the system control over time $[51,76]$. On the other hand, the adaptive decoders need to follow the nonstationary neural activities in order to improve the performance of BMI systems [77, 78]. The coadaptive BMI has later been presented as a novel architecture that goes beyond translational neural interface by merging with above two factors [51, 76, 79, 80]. Allowing brain and the intelligent decoder to adapt to each other during learning according to the task accomplishment, coadaptive BMI becomes attractive for the brain-controlled prosthesis in future clinical applications without requiring the real movements of the patients, for example, with tetraplegia.

3.2. Neural Coding for Human BMI. Similar to the coding methods used in animal models, the brain electrical signals used in BMI systems for human beings are mainly ECoG and scalp EEG. Scalp EEG (referred as EEG later) can be noninvasively detected from the skin surface according to the 10-20 system for positioning the electrodes, with the commonly adopted numbers of 32 and 64 channels for the whole brain recording, or according to 10-5 system for high-density EEG with 128 channels [81]. In comparison with another biosignal captured from the skin surface, EMG $(50 \mu \mathrm{V}-$ $10 \mathrm{mV})$, the amplitude of EEG $(10-20 \mu \mathrm{V})$ is much smaller and easily contaminated by head and neck muscle contractions, as well as artifacts, for example, eye blinks. EEG signals have been proposed for identifying neural instructions. For example, it has been widely known that the motion planning is associated with a decrease in EEG energy in the mu rhythm $(8-12 \mathrm{~Hz})$ over sensorimotor cortex, that is, eventrelated desynchronization (ERD), and after the execution of a motion there will be a rebound in the EEG power around $20 \mathrm{~Hz}$, that is, event-related synchronization (ERS) [82]. EEG-based BMI systems have been successfully applied on external device control by people with severe motor disorders, such as spinal cord injury (SCI) and muscular dystrophies [83, 84]. However, most of them showed little effect on motor recovery for stroke rehabilitation as pointed out in the reviews of Belda-Lois et al. and Mattia et al. [85, 86]. One of the major reasons is that, different from subjects with an intact brain (e.g., SCI), individual stroke survivors have varied brain lesion sites and sizes, which increases the difficulty of recognizing the correct motion patterns for each. The second reason could be that stroke patients are suffered from involuntary muscle hypertonia more often than SCI patients [87], and it would introduce extra noises to the EEG signals recorded. The third reason might be associated with the method of whole brain EEG recording. Once the learning capability of a BMI algorithm is powerful and easy to converge to a classified pattern with redundant EEG channel information (or even with repeatable patterns of noise, like EMG), the effort from the other side of neuroplasticity in the brain will be weakened. The reported pattern recognition rates of BMI with EEG for stroke varied greatly (e.g., from $60 \%$ to $90 \%$ [88]), and usually are lower than those for SCI. Therefore, more effective and accurate neural indicators from the brain are needed for human BMI design, especially in stroke rehabilitation.

Different from the skin surface electrodes of EEG, electrode grids that acquire ECoG can be placed subdurally (i.e., below the dura mater) or epidurally (i.e., on top of the dura) directly on the surface of the brain (i.e., subdural recordings) or on top of the dura (i.e., epidural recordings). Hence, ECoG signals are mesoscale activity of ensembles of neurons, which lie in the continuum between microscale single-unit action potential firings recorded intracortically and to macroscale EEG from the surface of the scalp. In fact, the rapidly growing interest in ECoG is mostly due to its improved signal characteristics relative to the artifact prone EEG. Compared with EEG, ECoG has finer spatial resolution (mesoscale (millimeters) versus macroscale (centimeters)) [89-91], broader spectral range $(0-500 \mathrm{~Hz}$ versus $0-40 \mathrm{~Hz})$ [92], higher amplitude (i.e., $50-100 \mu \mathrm{V}$ versus $10-20 \mu \mathrm{V}$ ) [93], and less vulnerability to movement artifacts $[5,93$, 94]. Moreover, ECoG electrode grids, which are typically platinum electrodes $4 \mathrm{~mm}$ ( $2.3 \mathrm{~mm}$ exposed) in diameter and are configured in either a grid (e.g., $8 \times 8$ electrodes) or strip (e.g., 4 or 6 electrodes) configuration with an interelectrode distance of usually $10 \mathrm{~mm}$, are far more likely to yield long-term functional stability [95-99] than intracortical electrodes, which induce complex histological responses that may impair neuronal recordings [100-102]. In fact, recent studies in primates demonstrated that the signal-to-noise ratio of ECoG signals is stable over several months [103]. Moreover these studies showed that cortical representations of three dimensional arm and joint movements that can be identified [7] and cortical control of three dimensional cursors were achieved [104] and maintained over several months.

However, since the placement of ECoG grid electrodes requires an invasive procedure (craniotomy and in most cases an incision to the dura), most ECoG-based human studies have recruited patients that were implanted as part of brain surgery to excise epileptic focus or mass lesion. Hence, most of the earlier ECoG-based studies often studied behaviors that were relevant to clinical evaluation of these patient populations, such as functional mapping of motor function. These early efforts culminated in the first comprehensive characterization of ECoG responses to visuomotor tasks in the late 1990s [105]. This has led to the appreciation of taskrelated modulations in high gamma $(70-200 \mathrm{~Hz})$ activity. The spatiotemporal patterns of these modulations are consistent sensorimotor function and its functional anatomy [106]. In recent years, however, ECoG has proved to be a vibrant recording technique for studying higher order functions 
$[6,107]$ and for brain-computer interfaces $[108,109]$ bringing together clinicians, neuroscientists, and engineers in the process. Moreover, epidural studies in animals [104, 110] corroborate the viability of epidural signals as a practical and less invasive signal modality, which can significantly reduce the risks of inflammations and complications.

The first use of ECoG as a practical and robust platform for translational applications beyond epileptology in human beings was demonstrated by Wang et al. [111] with an individual with tetraplegia caused by $\mathrm{C} 4$ level spinal cord injury. ECoG signals were recorded over the left sensorimotor cortex using a 32-contact high-density grid. The participant achieved robust volitional control of 3D cursor movement and a robotic arm. The participant was able to modulate his sensorimotor cortex with distinctive cortical activity patterns for different segments of the upper limb. The grid remained implanted for 28 days and did not cause any adverse effects. Another study by Hirata et al. showed control of a robotic arm in patients with moderate motor dysfunction due to stroke [112]. However, the effects of this training on rehabilitation in ipsilesional brain areas have not been recorded.

ECoG as a signal modality has also lent itself to uncovering potential signal features that could be exploited for hemispheric stroke. In recent years, there has been increased interest in how ipsilateral motor and motor-related areas activate in same-sided movements in both healthy and strokeaffected subjects [113-115]. These findings have motivated further explorations of whether ipsilateral activity in unaffected hemispheres could be used in neuroprosthetic applications for stroke-induced hemiparesis. Wisneski et al. [116] utilized ECoG recordings to comprehensively define ipsilateral physiology in motor-intact patients undergoing invasive monitoring. Electrocorticographic signals were recorded while the subjects engaged in ipsilateral and contralateral hand motor tasks. Ipsilateral hand movements were associated with low-frequency modulations (around $37.5 \mathrm{~Hz}$ ) in premotor cortex $\sim 160 \mathrm{~ms}$ before than activity related to contralateral hand movements. The authors therefore hypothesized that ipsilateral cortical activity is involved in motor planning (rather than execution). More recent studies $[8,117]$ have demonstrated that the ipsilateral cortical signals could be used to decode the direction of the joystick movement. Overall, these studies suggest that in motor-intact human subjects, ipsilateral activity during hand and arm movement is distinguishable from contralateral activity, is involved in planning rather than execution, and can be used as a viable control signal in BMIs [116]. Moreover, the fact that the premotor cortex control signal is in the low-frequency ranges highly suggests that ipsilateral (and contralesional) EEG signals could be used with patients with stroke for BMI control and possibly rehabilitation. In fact, functional imaging has demonstrated increased activity in the premotor cortices of motor-impaired stroke in unaffected hemispheres [118, 119]. This heightened activity could be a result of upregulation of motor planning due to the inability of executing the planned movement [116]. In a recent study Bundy et al. tested whether this heightened activity could be detected with an EEG-based BMI and converted into the desired action [88]. They recorded EEG signals from four chronic hemispheric stroke patients as they attempted real and imagined hand tasks using either their affected or unaffected hand. Low-frequency ipsilateral motor signals in the unaffected hemisphere, distinguishable from contralateral signals, were identified and subsequently used for a simple online BMI control task. They demonstrated that EEG signals from the unaffected hemisphere, associated with imagined movements of the affected hand, enabled stroke patients to control a cursor in one dimension. There is significant potential for this approach to be used as a novel tool for rehabilitation by slowly disengaging the unaffected hemisphere and engaging the affected hemisphere during BMI control.

\section{Intelligent Rehabilitation Robots Based on EMG Coding}

Intelligent rehabilitation robots usually refer to the systems that can interact with the voluntary motor intentions of a user. Besides the BMI technology introduced above, EMGcontrolled robotic system is another choice for rehabilitation, mainly due to the easy-access of the signal from the skin surface of a muscle. In this part, an overview of the rehabilitation strategies in recent robots was introduced first, and it was followed with a review on some latest representative EMG-controlled rehabilitation robots and their clinical applications.

4.1. Rehabilitation Strategies in Robots. The aims of the treatment in neural rehabilitation are mainly to rebuild the lost sensorimotor functions due to nervous system injuries, such as stroke, and to minimize the related paretic symptoms. The recovery in the rehabilitation is a motor relearning process; that is, the lost functions can be regained and maximized by intensive and repeated voluntary practices [120,121], and this concept has been applied in the traditional rehabilitation for decades.

Treatments in the rehabilitation are arduous processes. Training programs are usually time-consuming and laborintensive for both the therapist and the patient in one-to-one manual interaction. In these situations, rehabilitation robots have acted as the assistance to therapists, providing safe and intensive physical training with repeated motions [122128]. The most commonly reported motion types provided by developed rehabilitation robots are (1) continuous passive motion (CPM), (2) active-assisted movements, and (3) challenge-based movement. In treatments with continuous passive motion, the movements of the patient's limb(s) in the paretic side are guided by the robot system as the patient stays in a relaxed condition. This type of intervention was found to be effective in temporarily reducing muscular hypertonia and maintaining the flexibility of joints for stroke and spinal cord injury [129]; however, it contributed little to a permanent motor recovery in the central nervous system after stroke $[129,130]$. In active-assisted robotic treatment (or interactive robotic treatment), the rehabilitation robot provides external assisting forces when the patient can not complete a desired movement independently [123, 131, 132]. In this type of physical training, the robot first needs to identify the motor 

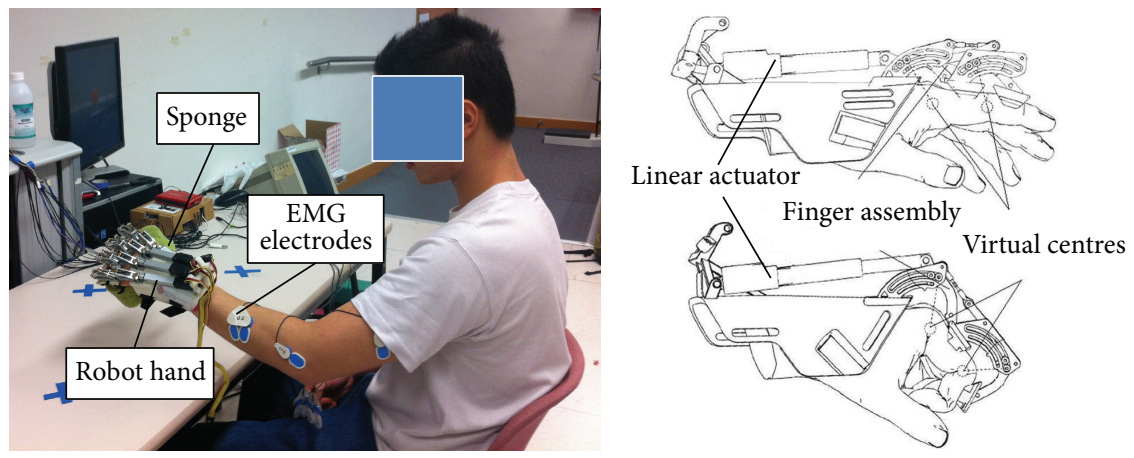

(a)

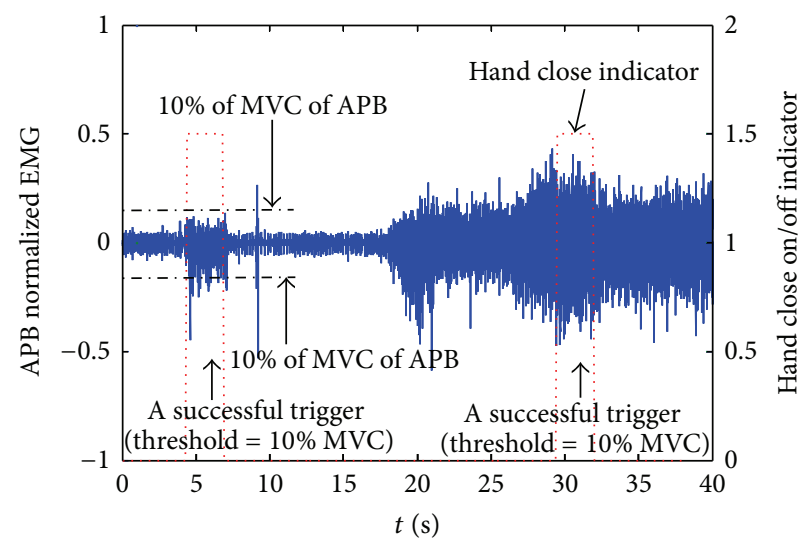

(b)

FigurE 5: (a) The EMG-triggered robot hand for upper limb training and (b) the representative EMG-triggered cycles for hand close of the robot $[10]$.

intention from the patient and then provides the interactive assistance to the paralyzed limb. This type of training has been found to be more effective in motor improvement than CPM in stroke rehabilitation [129]. Robotic treatment with challenge-based movement can assign training tasks with varied difficulty levels [124, 133, 134], which is effective in promotion of voluntary efforts from the patient according to the recovery progress. Active-assisted and challenge-based robotic training can be combined in one treatment to achieve a maximized motor recovery, and the key to a successful rehabilitation is the accurate interpretation of the voluntary motor intention of a user.

4.2. EMG-Controlled Rehabilitation Robots. EMG is the electricity generated in muscles under the control of the nervous systems. When an action potential transmitted from a motor neuron axon to the muscle fibers, a motor unit action potential is evoked. In comparison with the amplitude of neuronal signals, EMG's amplitude is much higher (usually in millivolt) even when detecting from the skin surface [9]. Therefore, EMG is a favorable biosignal to represent a user's voluntary motor intention in robotic design. For interpretation of EMG in the real-time control of robots, there are basically two methods, triggered mode and continuous mode. In the triggered mode, EMG was used to initiate the movement of the robot, and after that, the robot would work in a CPM mode $[10,123,132]$. A preset threshold for detection of the onset of EMG can be used to trigger the motion of the robot, for example, the EMG-triggered hand robot for upper limb training after stroke introduced in the study of $\mathrm{Hu}$ et al., as shown in Figure 5(a) [10]. The robot hand could help a stroke patient perform the hand close/open motions triggered by the residual EMG detected from the abductor pollicis brevis (APB) in the paretic side for controlling the hand close and the extensor digitorum (ED) for the hand open. Figure 5(b) shows the representative triggering cycles in the robot hand for hand close with the EMG from the APB muscle, where the triggering threshold was set at $10 \%$ of the EMG amplitude when conducting the maximal voluntary contraction (MVC) [10]. Once the realtime EMG amplitude was above the threshold and kept for 3 seconds, the robot hand would perform hand close motion with a constant angular velocity of $22^{\circ} / \mathrm{s}$ with the virtual center of the metacarpophalangeal (MCP) joints and $26^{\circ} / \mathrm{s}$ at that of proximal interphalangeal (PIP) joints. By using this EMG-triggered robot hand, a pilot clinical trial of upper limb training on ten subjects with chronic stroke was conducted, and each of the subjects received 20 training sessions with an intensity of 3-5 sessions/week. After the training, it was found that the robot hand assisted rehabilitation could significantly improve the finger functions and the muscle coordination in the whole upper limb [10]. 

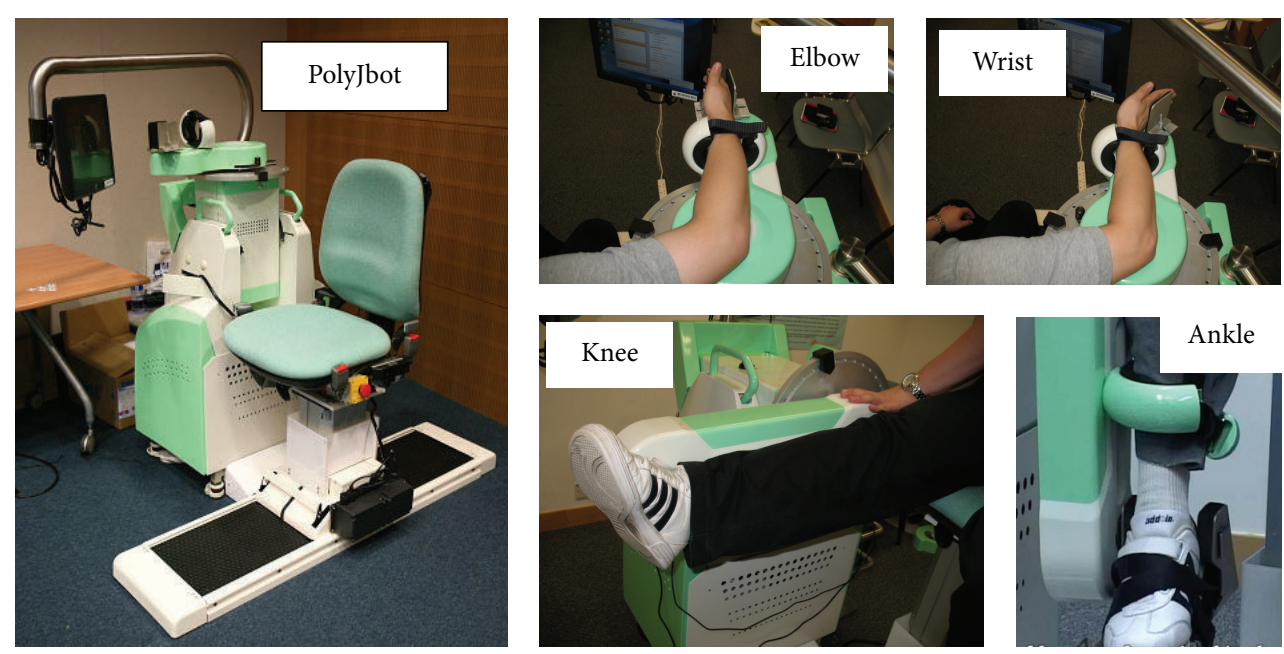

Figure 6: The continuous EMG-driven robot (PolyJbot) for joint training at the elbow, the wrist, the knee, and the ankle [130, 135, 136].

In a continuous EMG-driven robot system, the behaviors of the robot are controlled by the continuous variation of EMG, which requires the user to generate desired EMG patterns to instruct the movement of the robot [135], for example, the continuous EMG-driven robotic system (PolyJbot) developed for multijoint training by Tong's group shown in Figure $6[130,136,137]$. The robot can provide treatments on the elbow, the wrist, the knee, and the ankle with a continuous EMG-controlled algorithm. During the training, a subject needs to conduct joint extension and flexion by tracing a target cursor on the screen, and the robot will provide assistive torque to the joint, which is proportional to the EMG amplitude of a target muscle, for example, extensor carpi radialis (ECR) in wrist extension. In the algorithm design, the more muscle effort generated, the more assistive torque obtained with an attempt to maximize the voluntary effort during the training. In comparison with the training effects by robot-assisted CPM mode, the continuous EMGdriven mode could achieve more significant improvements in the release of muscle spasticity (i.e., hypertonia) and improvement of muscle coordination in the wrist joint, as well as in the shoulder/elbow part [130, 137]. Furthermore, the motor outcomes gained after the continuous EMG-driven robot-assisted training could be maintained for 3 months [130].

EMG not only can be applied as the controlling signal in rehabilitation robots, but also has been applied on quantitative evaluation of the rehabilitation effects complementary to the subjective clinical assessment tools used in routine practice. For example, the coordination among muscles could be measured by EMG phasic change in muscle pairs $[10,130$, 136-138]. The extent of the cocontraction phase was quantitatively evaluated by a cocontraction index (CI) between the EMG trials of two muscles. The CI values could be used to monitor the recovery progress in muscle coordination during the robot-assisted rehabilitation. Figure 7 shows an example of the calculated CIs of the muscles in the upper limb during PolyJbot assisted poststroke wrist training in different sessions [137]. A decrease in the CI values usually was related to a release of muscle spasticity and more independent contraction of the muscle pair, that is, better coordination.

\section{Future Prospects and Conclusions}

Modern neural rehabilitation heavily relies on the advances of neural computational techniques in diagnosis, treatment, and evaluation, which promise the future rehabilitation to be more automatic, economical, and convenient. Instead of receiving the treatments in hospitals or medical centers, future rehabilitation will be mainly home-based and subjectcustomized training with telecommunication for evaluation and follow-up to meet the fast growing market of home health care services [139]. To achieve this, further investigations are needed in neural coding techniques mainly in the following aspects.

(1) Reduction of the calculation cost: high accuracy usually is sacrificed with the cost of calculation efficiency. Smart computational methods are needed to locate the areas of interest in neural imaging and highly relevant channels in BMI systems for individual subjects, with necessary accuracy for a real-time system. Currently, multichannel EEG systems are used for both invasive and noninvasive BMI systems, which are associated with large amounts of data to be explored. Effective channel selection methods with prioritized channel information should be useful to lower down the calculation cost, as some pioneers reported in the literature [140, 141]. More efforts are needed for the investigation of the long-term rehabilitation programs associated with the variation of neural plasticity in individuals, like persons after stroke who have varied brain lesions.

(2) Comparative study between scalp EEG and ECoG: ECoG has higher resolution and signal quality than scalp EEG. However, the invasiveness of ECoG as a recording modality limits its usage. Modeling and 

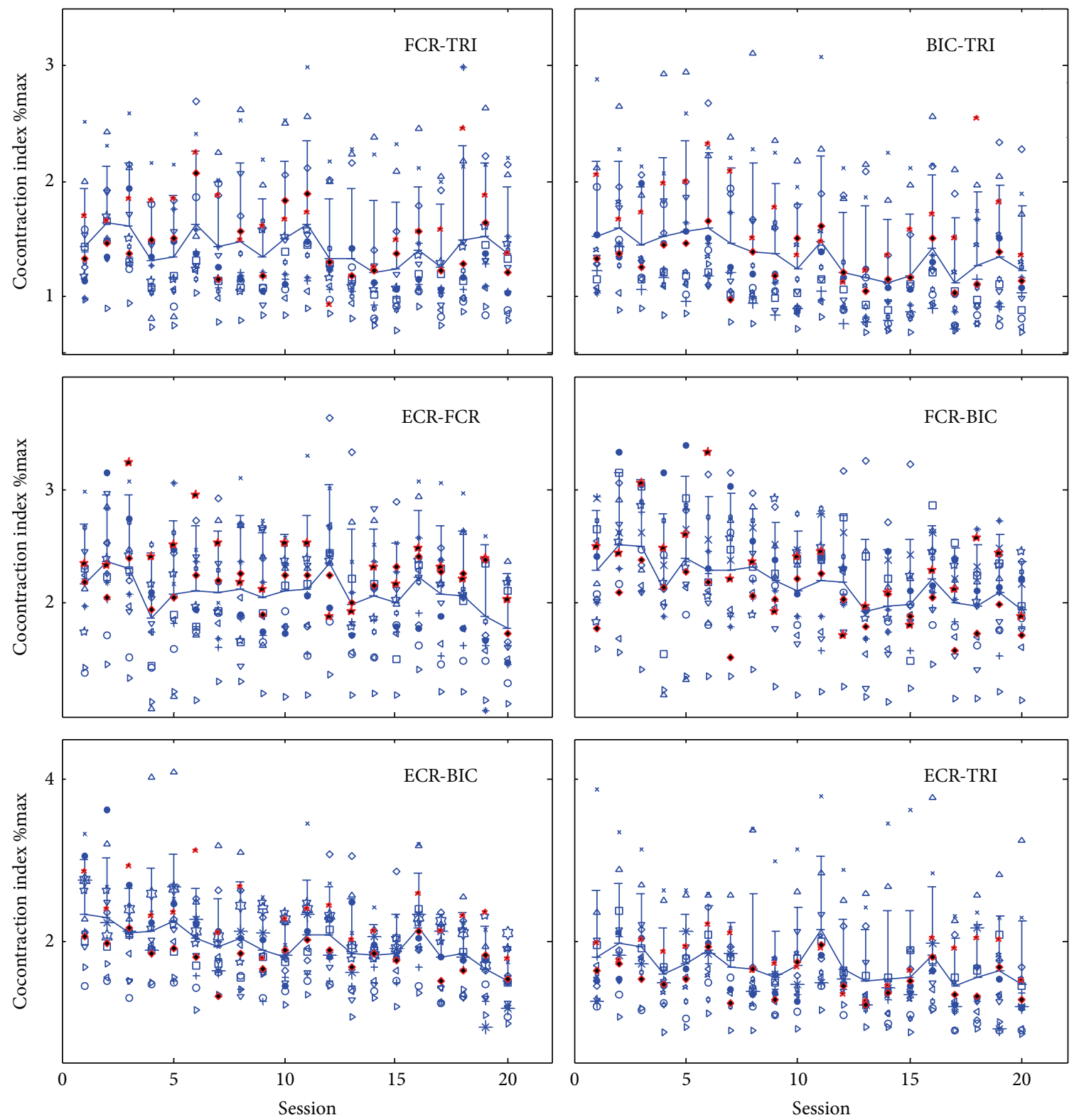

$\begin{array}{ll}-\mathrm{S} 1 & \Delta \mathrm{S} 9 \\ \circ \mathrm{S} 2 & \triangleleft \mathrm{S} 10 \\ \times \mathrm{S} 3 & \triangleright \mathrm{S} 11 \\ +\mathrm{S} 4 & \star \mathrm{S} 12 \\ * \mathrm{~S} 5 & \bullet \mathrm{S} 13 \\ -\mathrm{S} 6 & \star \mathrm{S} 14 \\ \bullet \mathrm{S} 7 & * \mathrm{~S} 15 \\ -\mathrm{S} 8 & \end{array}$

$\begin{array}{ll}\bullet \mathrm{S} 1 & \Delta \mathrm{S} 9 \\ \circ \mathrm{S} 2 & \triangleleft \mathrm{S} 10 \\ \times \mathrm{S} 3 & \triangleright \mathrm{S} 11 \\ +\mathrm{S} 4 & \star \mathrm{S} 12 \\ * \mathrm{~S} 5 & \bullet \mathrm{S} 13 \\ \square \mathrm{S} 6 & \star \mathrm{S} 14 \\ \bullet \mathrm{S} 7 & \bullet \mathrm{S} 15 \\ -\mathrm{S} 8 & \end{array}$

Figure 7: The cocontraction indexes between different muscle pairs during the continuous EMG-driven robot-assisted wrist training on 15 subjects with chronic stroke (S1-S15) [136]. FCR, flexor carpi radialis; ECR, extensor carpi radialis; BIC, biceps brachii; TRI, triceps brachii. The cocontraction index has a range from 0 to 1 and represents the extent of cocontraction phase of a pair of muscles, in comparison with the maximum value of 1 . If both muscles contract at their maximum level at the same time, then the value will be 1 ; while if there is no cocontraction of the two muscles, the value will be zero [136]. 
source localization/projection studies could be helpful to investigate the relationships between ECoG and scalp EEG, which may lead to noninvasive EEG with needed resolution close to ECoG. For example, attempts have been made to compare the EEG and ECoG in persons with epilepsy [142]. However, more intensive investigations on the projection between EEG and ECoG are needed in other pathological and clinical applications in the future.

(3) Rehabilitation effectiveness of BMI systems: although BMI-training systems have been proposed for patients with neural disorders, for example, stroke, the rehabilitation effects are still questioned [143]. Stroke patients could use the system with a high recognition rates. However, this may not directly lead to an improvement in the paralyzed limb functions $[85,86]$. It is worthwhile to investigate whether redundant EEG channel information in the BMI system made the recognition task too easy to benefit the motor recovery.

(4) Easy and reliable bioparameters in system control and evaluation of recovery: it is necessary to utilize objective and quantitative evaluation methods for monitoring recovery progress in rehabilitation. However, the operations of most of current robots are not easy for patients to use them at home without supervision. Training systems should be much simplified, especially on the detection of key bioparameters for system control and evaluation in the home-based devices.

\section{Conflict of Interests}

No commercial party having a direct financial interest in the results of the research supporting this paper has or will confer a benefit upon the authors or upon any organization with which the authors are associated.

\section{Acknowledgments}

The authors acknowledge the financial supports: ITF grant (ITS/033/12) from the Innovation and Technology Commission of the Hong Kong Special Administrative Region; Natural Science Foundation of China (no. 61473261); Zhejiang provincial Natural Science Foundation of China (no. LY14F030015).

\section{References}

[1] X. Wu, B. Zhu, L. Fu et al., "Prevalence, incidence, and mortality of stroke in the chinese island populations: a systematic review," PLoS ONE, vol. 8, no. 11, Article ID e78629, 2013.

[2] Heart Disease and Stroke Statistics, American Heart Association, 2011, http://www.stroke.org/site/DocServer/STROKE_ 101_Fact_Sheet.pdf?docID $=4541$.

[3] Rehabilitation Service Industry Reports of 2013, First Research, Christchurch, New Zealand; Census Bureau U.S., Washington, DC, USA, 2013.
[4] M. B. Ahrens, J. M. Li, M. B. Orger et al., "Brain-wide neuronal dynamics during motor adaptation in zebrafish," Nature, vol. 485, no. 7399, pp. 471-477, 2012.

[5] W. J. Freeman, M. D. Holmes, B. C. Burke, and S. Vanhatalo, "Spatial spectra of scalp EEG and EMG from awake humans," Clinical Neurophysiology, vol. 114, no. 6, pp. 1053-1068, 2003.

[6] A. Gunduz, P. Brunner, A. Daitch et al., "Decoding covert spatial attention using electrocorticographic (ECoG) signals in humans," NeuroImage, vol. 60, no. 4, pp. 2285-2293, 2012.

[7] Z. C. Chao, Y. Nagasaka, and N. Fujii, "Long-term asynchronous decoding of arm motion using electrocorticographic signals in monkey," Frontiers in Neuroengineering, vol. 3, article 3, 2010.

[8] K. Ganguly, L. Secundo, G. Ranade et al., "Cortical representation of ipsilateral arm movements in monkey and man," Journal of Neuroscience, vol. 29, no. 41, pp. 12948-12956, 2009.

[9] K. Nishihara and T. Isho, "Location of electrodes in surface EMG," in EMG Methods for Evaluating Muscle and Nerve Function, M. Schwartz, Ed., InTech, 2012.

[10] X. L. Hu, K. Y. Tong, X. J. Wei, W. Rong, E. A. Susanto, and S. K. Ho, "The effects of post-stroke upper-limb training with an electromyography (EMG)-driven hand robot," Journal of Electromyography \& Kinesiology, vol. 23, no. 5, pp. 1065-1074, 2013.

[11] A. R. Fugl-Meyer, L. Jaasko, I. Leyman, S. Olsson, and S. Steglind, "The post stroke hemiplegic patient I: a method for evaluation of physical performance," Scandinavian Journal of Rehabilitation Medicine, vol. 7, no. 1, pp. 13-31, 1975.

[12] B. Ashworth, "Preliminary trials of carisoprodol in multiple sclerosis," The Practitioner, vol. 192, pp. 540-542, 1964.

[13] K. N. Kay, T. Naselaris, R. J. Prenger, and J. L. Gallant, "Identifying natural images from human brain activity," Nature, vol. 452, no. 7185, pp. 352-355, 2008.

[14] T. Naselaris, R. J. Prenger, K. N. Kay, M. Oliver, and J. L. Gallant, "Bayesian reconstruction of natural images from human brain activity," Neuron, vol. 63, no. 6, pp. 902-915, 2009.

[15] J. P. Gallivan, D. A. McLean, K. F. Valyear, and J. C. Culham, "Decoding the neural mechanisms of human tool use," eLife, vol. 2, Article ID e00425, 2013.

[16] T. M. Mitchell, S. V. Shinkareva, A. Carlson et al., "Predicting human brain activity associated with the meanings of nouns," Science, vol. 320, no. 5880, pp. 1191-1195, 2008.

[17] F. Pereira, T. Mitchell, and M. Botvinick, "Machine learning classifiers and fMRI: a tutorial overview," NeuroImage, vol. 45, no. 1, pp. S199-S209, 2009.

[18] C. Chu, Y. Ni, G. Tan, C. J. Saunders, and J. Ashburner, "Kernel regression for fMRI pattern prediction," NeuroImage, vol. 56, no. 2, pp. 662-673, 2011.

[19] R. Genuer, V. Michel, E. Eger, and B. Thirion, "Random Forests based feature selection for decoding fMRI data," in Proceedings of the 19th International Symposium on Computational Statistic (COMPSTAT'10), pp. 1-8, 2010.

[20] X. Shen and F. G. Meyer, "Low-dimensional embedding of fMRI datasets," NeuroImage, vol. 41, no. 3, pp. 886-902, 2008.

[21] S. M. LaConte, "Decoding fMRI brain states in real-time," NeuroImage, vol. 56, no. 2, pp. 440-454, 2011.

[22] D. Scheinost, M. Hampson, M. Qiu, J. Bhawnani, R. T. Constable, and X. Papademetris, "A graphics processing unit accelerated motion correction algorithm and modular system for real-time fMRI," Neuroinformatics, vol. 11, no. 3, pp. 291-300, 2013. 
[23] T. Schrödel, R. Prevedel, K. Aumayr, M. Zimmer, and A. Vaziri, "Brain-wide 3D imaging of neuronal activity in Caenorhabditis elegans with sculpted light," Nature Methods, vol. 10, pp. 10131020, 2013.

[24] J. Li, K.-X. Huang, and W.-D. Le, "Establishing a novel C. elegans model to investigate the role of autophagy in amyotrophic lateral sclerosis," Acta Pharmacologica Sinica, vol. 34, no. 5, pp. 644-650, 2013.

[25] H. Kang and J. W. Lichtman, "Motor axon regeneration and muscle reinnervation in young adult and aged animals," The Journal of Neuroscience, vol. 33, no. 50, pp. 19480-19491, 2013.

[26] D. Sheng, D. Qu, K. H. H. Kwok et al., "Deletion of the WD40 domain of LRRK2 in zebrafish causes parkinsonism-like loss of neurons and locomotive defect," PLoS Genetics, vol. 6, no. 4, 2010.

[27] L. Luo, E. M. Callaway, and K. Svoboda, "Genetic dissection of neural circuits,” Neuron, vol. 57, no. 5, pp. 634-660, 2008.

[28] J. J. Mancuso, J. Kim, S. Lee, S. Tsuda, N. B. H. Chow, and G. J. Augustine, "Optogenetic probing of functional brain circuitry," Experimental Physiology, vol. 96, no. 1, pp. 26-33, 2010.

[29] L. Lin, R. Osan, S. Shoham, W. Jin, W. Zuo, and J. Z. Tsien, "Identification of network-level coding units for real-time representation of episodic experiences in the hippocampus," Proceedings of the National Academy of Sciences of the United States of America, vol. 102, no. 17, pp. 6125-6130, 2005.

[30] L. Lin, G. Chen, K. Xie, K. A. Zaia, S. Zhang, and J. Z. Tsien, "Large-scale neural ensemble recording in the brains of freely behaving mice," Journal of Neuroscience Methods, vol. 155, no. 1, pp. 28-38, 2006.

[31] A. Joudaki, N. Salehi, M. Jalili, and M. G. Knyazeva, "EEG-based functional brain networks: does the network size matter?" PLoS ONE, vol. 7, no. 4, Article ID e35673, 2012.

[32] M. D. Greicius, K. Supekar, V. Menon, and R. F. Dougherty, "Resting-state functional connectivity reflects structural connectivity in the default mode network," Cerebral Cortex, vol. 19, no. 1, pp. 72-78, 2009.

[33] A. Venkataraman, K. R. A. van Dijk, R. L. Buckner, and P. Golland, "Exploring functional connectivity in fMRI via clustering," in Proceedings of the IEEE International Conference on Acoustics, Speech, and Signal Processing (ICASSP 2009), pp. 441-444, April 2009.

[34] M. E. Thomason, M. T. Dassanayake, S. Shen et al., "Crosshemispheric functional connectivity in the human fetal brain," Science Translational Medicine, vol. 5, no. 173, Article ID 173ra24, 2013.

[35] J. Sun, X. Hu, X. Huang et al., "Inferring consistent functional interaction patterns from natural stimulus FMRI data," NeuroImage, vol. 61, no. 4, pp. 987-999, 2012.

[36] A. W. Toga, K. A. Clark, P. M. Thompson, D. W. Shattuck, and J. D. van Horn, "Mapping the human connectome," Neurosurgery, vol. 71, no. 1, pp. 1-5, 2012.

[37] S. W. Oh, J. A. Harris, L. Ng et al., "A mesoscale connectome of the mouse brain," Nature, vol. 508, pp. 207-214, 2014.

[38] J. Kim, T. Zhao, R. S. Petralia et al., "mGRASP enables mapping mammalian synaptic connectivity with light microscopy," Nature Methods, vol. 9, no. 1, pp. 96-102, 2012.

[39] S.-Y. Takemura, A. Bharioke, Z. Lu et al., "A visual motion detection circuit suggested by Drosophila connectomics," Nature, vol. 500, no. 7461, pp. 175-181, 2013.

[40] C. Eliasmith, T. C. Stewart, X. Choo et al., "A large-scale model of the functioning brain," Science, vol. 338, no. 6111, pp. 12021205, 2012.
[41] T. Geijtenbeek, M. van de Panne, and F. van der Stappen, "Flexible muscle-based locomotion for bipedal creatures," in ACM Transactions on Graphics, 2013.

[42] J. L. Collinger, B. Wodlinger, J. E. Downey et al., "Highperformance neuroprosthetic control by an individual with tetraplegia," The Lancet, vol. 381, no. 9866, pp. 557-564, 2013.

[43] J. K. Chapin, K. A. Moxon, R. S. Markowitz, and M. A. L. Nicolelis, "Real-time control of a robot arm using simultaneously recorded neurons in the motor cortex," Nature Neuroscience, vol. 2, no. 7, pp. 664-670, 1999.

[44] C. Ethier, E. R. Oby, M. J. Bauman, and L. E. Miller, "Restoration of grasp following paralysis through brain-controlled stimulation of muscles," Nature, vol. 485, no. 7398, pp. 368-371, 2012.

[45] L. R. Hochberg, D. Bacher, B. Jarosiewicz et al., "Reach and grasp by people with tetraplegia using a neurally controlled robotic arm," Nature, vol. 485, no. 7398, pp. 372-375, 2012.

[46] C. T. Moritz, S. I. Perlmutter, and E. E. Fetz, "Direct control of paralysed muscles by cortical neurons," Nature, vol. 456, no. 7222, pp. 639-642, 2008.

[47] S. Musallam, B. D. Corneil, B. Greger, H. Scherberger, and R. A. Andersen, "Cognitive control signals for neural prosthetics," Science, vol. 305, no. 5681, pp. 258-262, 2004.

[48] J. E. O’Doherty, M. A. Lebedev, P. J. Ifft et al., "Active tactile exploration using a brain-machine-brain interface," Nature, vol. 479, no. 7372, pp. 228-231, 2011.

[49] G. Santhanam, S. I. Ryu, B. M. Yu, A. Afshar, and K. V. Shenoy, "A high-performance brain-computer interface," Nature, vol. 442, no. 7099, pp. 195-198, 2006.

[50] M. D. Serruya, N. G. Hatsopoulos, L. Paninski, M. R. Fellows, and J. P. Donoghue, "Instant neural control of a movement signal," Nature, vol. 416, no. 6877, pp. 141-142, 2002.

[51] D. M. Taylor, S. I. H. Tillery, and A. B. Schwartz, "Direct cortical control of 3D neuroprosthetic devices," Science, vol. 296, no. 5574, pp. 1829-1832, 2002.

[52] C. E. Vargas-Irwin, G. Shakhnarovich, P. Yadollahpour, J. M. K. Mislow, M. J. Black, and J. P. Donoghue, "Decoding complete reach and grasp actions from local primary motor cortex populations," The Journal of Neuroscience, vol. 30, no. 29, pp. 9659-9669, 2010.

[53] M. Velliste, S. Perel, M. C. Spalding, A. S. Whitford, and A. B. Schwartz, "Cortical control of a prosthetic arm for self-feeding," Nature, vol. 453, no. 7198, pp. 1098-1101, 2008.

[54] J. Wessberg, C. R. Stambaugh, J. D. Kralik et al., "Real-time prediction of hand trajectory by ensembles of cortical neurons in primates," Nature, vol. 408, no. 6810, pp. 361-365, 2000.

[55] G. A. Orban, D. van Essen, and W. Vanduffel, "Comparative mapping of higher visual areas in monkeys and humans," Trends in Cognitive Sciences, vol. 8, no. 7, pp. 315-324, 2004.

[56] A. P. Georgopoulos, J. F. Kalaska, and R. Caminiti, "On the relations between the direction of two-dimensional arm movements and cell discharge in primate motor cortex," The Journal of Neuroscience, vol. 2, no. 11, pp. 1527-1537, 1982.

[57] A. K. Bansal, W. Truccolo, C. E. Vargas-Irwin, and J. P. Donoghue, "Decoding 3D reach and grasp from hybrid signals in motor and premotor cortices: spikes, multiunit activity, and local field potentials," Journal of Neurophysiology, vol. 107, no. 5, pp. 1337-1355, 2012.

[58] J. M. Carmena, M. A. Lebedev, R. E. Crist et al., "Learning to control a brain-machine interface for reaching and grasping by primates," PLoS Biology, vol. 1, no. 2, article E42, 2003. 
[59] M. A. Lebedev, J. M. Carmena, J. E. O’Doherty et al., "Cortical ensemble adaptation to represent velocity of an artificial actuator controlled by a brain-machine interface," The Journal of Neuroscience, vol. 25, no. 19, pp. 4681-4693, 2005.

[60] S.-P. Kim, J. C. Sanchez, D. Erdogmus et al., "Divide-andconquer approach for brain machine interfaces: nonlinear mixture of competitive linear models," Neural Networks, vol. 16, no. 5-6, pp. 865-871, 2003.

[61] A. E. Brockwell, A. L. Rojas, and R. E. Kass, "Recursive Bayesian decoding of motor cortical signals by particle filtering," Journal of Neurophysiology, vol. 91, no. 4, pp. 1899-1907, 2004.

[62] W. Wu, Y. Gao, E. Bienenstock, J. P. Donoghue, and M. J. Black, "Bayesian population decoding of motor cortical activity using a Kalman filter," Neural Computation, vol. 18, no. 1, pp. 80-118, 2006.

[63] K. Doya, S. Ishii, A. Pouget, and R. Rao, Bayesian Brain: Probabilistic Approaches to Neural Coding, MIT Press, 2007.

[64] A. Ergun, R. Barbieri, U. T. Eden, M. A. Wilson, and E. N. Brown, "Construction of point process adaptive filter algorithms for neural systems using sequential Monte Carlo methods," IEEE Transactions on Biomedical Engineering, vol. 54, pp. 419-428, 2007.

[65] Y. Wang, A. R. C. Paiva, J. C. Príncipe, and J. C. Sanchez, "Sequential Monte Carlo point-process estimation of kinematics from neural spiking activity for brain-machine interfaces," Neural Computation, vol. 21, no. 10, pp. 2894-2930, 2009.

[66] W. Truccolo, U. T. Eden, M. R. Fellows, J. P. Donoghue, and E. N. Brown, "A point process framework for relating neural spiking activity to spiking history, neural ensemble, and extrinsic covariate effects," Journal of Neurophysiology, vol. 93, no. 2, pp. 1074-1089, 2005.

[67] Y. Wang and J. C. Principe, "Instantaneous estimation of motor cortical neural encoding for online brain-machine interfaces," Journal of Neural Engineering, vol. 7, no. 5, Article ID 056010, 2010.

[68] J. C. Sanchez, J. M. Carmena, M. A. Lebedev, M. A. L. Nicolelis, J. G. Harris, and J. C. Principe, "Ascertaining the importance of neurons to develop better brain-machine interfaces," IEEE Transactions on Biomedical Engineering, vol. 51, no. 6, pp. 943953, 2004.

[69] Y. Wang, J. C. Principe, and J. C. Sanchez, "Ascertaining neuron importance by information theoretical analysis in motor BrainMachine Interfaces," Neural Networks, vol. 22, no. 5-6, pp. 781790, 2009.

[70] K. Xu, Y. Wang, Y. Wang et al., "Local-learning-based neuron selection for grasping gesture prediction in motor brain machine interfaces," Journal of Neural Engineering, vol. 10, no. 2, Article ID 026008, 2013.

[71] M. A. Lebedev and M. A. L. Nicolelis, "Brain-machine interfaces: past, present and future," Trends in Neurosciences, vol. 29, no. 9, pp. 536-546, 2006.

[72] F. A. Mussa-Ivaldi, S. T. Alford, M. Chiappalone et al., "New perspectives on the dialogue between brains and machines," Frontiers in Neuroscience, vol. 4, no. 1, pp. 44-52, 2010.

[73] O. Yizhar, L. E. Fenno, T. J. Davidson, M. Mogri, and K. Deisseroth, "Optogenetics in neural systems," Neuron, vol. 71, no. 1, pp. 9-34, 2011.

[74] A. M. Aravanis, L.-P. Wang, F. Zhang et al., "An optical neural interface: in vivo control of rodent motor cortex with integrated fiberoptic and optogenetic technology," Journal of Neural Engineering, vol. 4, no. 3, pp. S143-S156, 2007.
[75] X. Han, B. Y. Chow, H. Zhou et al., "A high-light sensitivity optical neural silencer: development and application to optogenetic control of non-human primate cortex," Frontiers in Systems Neuroscience, vol. 13, article 18, 2011.

[76] G. J. Gage, K. A. Ludwig, K. J. Otto, E. L. Ionides, and D. R. Kipke, "Naïve coadaptive cortical control," Journal of Neural Engineering, vol. 2, no. 2, pp. 52-63, 2005.

[77] Z. Li, J. E. O’Doherty, M. A. Lebedev, and M. A. Nicolelis, “Adaptive decoding for brain-machine interfaces through Bayesian parameter updates," Neural Computation, vol. 23, no. 12, pp. 3162-3204, 2011.

[78] W. Wu and N. G. Hatsopoulos, "Real-time decoding of nonstationary neural activity in motor cortex," IEEE Transactions on Neural Systems and Rehabilitation Engineering, vol. 16, no. 3, pp. 213-222, 2008.

[79] J. DiGiovanna, B. Mahmoudi, J. Fortes, J. C. Principe, and J. C. Sanchez, "Coadaptive brain-machine interface via reinforcement learning," IEEE Transactions on Biomedical Engineering, vol. 56, no. 1, pp. 54-64, 2009.

[80] B. Mahmoudi and J. C. Sanchez, "A symbiotic brain-machine interface through value-based decision making," PLoS ONE, vol. 6, no. 3, Article ID e14760, 2011.

[81] V. Jurcak, D. Tsuzuki, and I. Dan, "10/20, 10/10, and 10/5 systems revisited: their validity as relative head-surface-based positioning systems," NeuroImage, vol. 34, no. 4, pp. 1600-1611, 2007.

[82] S. R. Soekadar, M. Witkowski, J. Mellinger, A. Ramos, N. Birbaumer, and L. G. Cohen, "ERD-based online brain-machine interfaces (BMI) in the context of neurorehabilitation: optimizing BMI learning and performance," IEEE Transactions on Neural Systems and Rehabilitation Engineering, vol. 19, no. 5, pp. 542-549, 2011.

[83] N. Birbaumer and L. G. Cohen, "Brain-computer interfaces: communication and restoration of movement in paralysis," The Journal of Physiology, vol. 579, no. 3, pp. 621-636, 2007.

[84] J. R. Wolpaw, "Brain-computer interfaces as new brain output pathways," Journal of Physiology, vol. 579, no. 3, pp. 613-619, 2007.

[85] J.-M. Belda-Lois, S. Mena-del Horno, I. Bermejo-Bosch et al., "Rehabilitation of gait after stroke: a review towards a top-down approach," Journal of NeuroEngineering and Rehabilitation, vol. 8, no. 1, article 66, 2011.

[86] D. Mattia, F. Pichiorri, M. Molinari, and R. Rupp, "Brain computer interface for hand motor function restoration and rehabilitation," in Towards Practical Brain-Computer Interfaces, B. Allison, Ed., Springer, Berlin, Germany, 2012.

[87] A. Thibaut, C. Chatelle, E. Ziegler, M.-A. Bruno, S. Laureys, and O. Gosseries, "Spasticity after stroke: physiology, assessment and treatment," Brain Injury, vol. 27, no. 10, pp. 1093-1105, 2013.

[88] D. T. Bundy, M. Wronkiewicz, M. Sharma, D. W. Moran, M. Corbetta, and E. C. Leuthardt, "Using ipsilateral motor signals in the unaffected cerebral hemisphere as a signal platform for brain-computer interfaces in hemiplegic stroke survivors," Journal of Neural Engineering, vol. 9, no. 3, Article ID 036011, 2012.

[89] W. J. Freeman, L. J. Rogers, M. D. Holmes, and D. L. Silbergeld, "Spatial spectral analysis of human electrocorticograms including the alpha and gamma bands," Journal of Neuroscience Methods, vol. 95, no. 2, pp. 111-121, 2000.

[90] E. C. Leuthardt, Z. Freudenberg, D. Bundy, and J. Roland, "Microscale recording from human motor cortex: implications 
for minimally invasive electrocorticographic brain-computer interfaces," Neurosurgical Focus, vol. 27, no. 1, article E10, 2009.

[91] M. W. Slutzky, L. R. Jordan, T. Krieg, M. Chen, D. J. Mogul, and L. E. Miller, "Optimal spacing of surface electrode arrays for brain-machine interface applications," Journal of Neural Engineering, vol. 7, no. 2, Article ID 026004, 2010.

[92] R. J. Staba, C. L. Wilson, A. Bragin, and I. Fried, "Quantitative analysis of high-frequency oscillations $(80-500 \mathrm{~Hz})$ recorded in human epileptic hippocampus and entorhinal cortex," Journal of Neurophysiology, vol. 88, no. 4, pp. 1743-1752, 2002.

[93] A. T. Valderrama, R. Oostenveld, M. J. Vansteensel, G. M. Huiskamp, and N. F. Ramsey, "Gain of the human dura in vivo and its effects on invasive brain signal feature detection," Journal of Neuroscience Methods, vol. 187, no. 2, pp. 270-279, 2010.

[94] T. Ball, E. Demandt, I. Mutschler et al., "Movement related activity in the high gamma range of the human EEG," NeuroImage, vol. 41, no. 2, pp. 302-310, 2008.

[95] L. A. Bullara, W. F. Agnew, T. G. H. Yuen, S. Jacques, and R. H. Pudenz, "Evaluation of electrode array material for neural prostheses," Neurosurgery, vol. 5, no. 6, pp. 681-686, 1979.

[96] G. E. Loeb, A. E. Walker, S. Uematsu, and B. W. Konigsmark, "Histological reaction to various conductive and dielectric films chronically implanted in the subdural space," Journal of Biomedical Materials Research, vol. 11, no. 2, pp. 195-210, 1977.

[97] E. Margalit, J. D. Weiland, R. E. Clatterbuck et al., "Visual and electrical evoked response recorded from subdural electrodes implanted above the visual cortex in normal dogs under two methods of anesthesia," Journal of Neuroscience Methods, vol. 123, no. 2, pp. 129-137, 2003.

[98] W. H. Pilcher and W. G. Rusyniak, "Complications of epilepsy surgery," Neurosurgery Clinics of North America, vol. 4, no. 2, pp. 311-325, 1993.

[99] T. G. H. Yuen, W. F. Agnew, and L. A. Bullara, "Tissue response to potential neuroprosthetic materials implanted subdurally," Biomaterials, vol. 8, no. 2, pp. 138-141, 1987.

[100] W. Shain, L. Spataro, J. Dilgen et al., "Controlling cellular reactive responses around neural prosthetic devices using peripheral and local intervention strategies," IEEE Transactions on Neural Systems and Rehabilitation Engineering, vol. 11, no. 2, pp. 186-188, 2003.

[101] J. Thelin, H. Jörntell, E. Psouni et al., "Implant size and fixation mode strongly influence tissue reactions in the CNS," PLoS ONE, vol. 6, no. 1, Article ID e16267, 2011.

[102] J. C. Williams, J. A. Hippensteel, J. Dilgen, W. Shain, and D. R. Kipke, "Complex impedance spectroscopy for monitoring tissue responses to inserted neural implants," Journal of Neural Engineering, vol. 4, no. 4, pp. 410-423, 2007.

[103] G. Schalk, "Can electrocorticography (ECoG) support robust and powerful brain-computer interfaces?" Frontiers in Neuroengineering, vol. 3, article 9, 2010.

[104] A. G. Rouse, J. J. Williams, J. J. Wheeler, and D. W. Moran, "Cortical adaptation to a chronic micro- electrocorticographic brain computer interface," The Journal of Neuroscience, vol. 33, no. 4, pp. 1326-1330, 2013.

[105] N. E. Crone, D. L. Miglioretti, B. Gordon, and R. P. Lesser, "Functional mapping of human sensorimotor cortex with electrocorticographic spectral analysis: II. Event-related synchronization in the gamma band," Brain, vol. 121, no. 12, pp. 23012315, 1998.

[106] K. J. Miller, E. C. Leuthardt, G. Schalk et al., "Spectral changes in cortical surface potentials during motor movement," The Journal of Neuroscience, vol. 27, no. 9, pp. 2424-2432, 2007.
[107] N. Mesgarani and E. F. Chang, "Selective cortical representation of attended speaker in multi-talker speech perception," Nature, vol. 484, no. 7397, pp. 233-236, 2012.

[108] E. C. Leuthardt, C. Gaona, M. Sharma et al., "Using the electrocorticographic speech network to control a brain-computer interface in humans," Journal of Neural Engineering, vol. 8, no. 3, Article ID 036004, 2011.

[109] E. C. Leuthardt, G. Schalk, J. R. Wolpaw, J. G. Ojemann, and D. W. Moran, "A brain-computer interface using electrocorticographic signals in humans," Journal of Neural Engineering, vol. 1, no. 2, pp. 63-71, 2004.

[110] R. D. Flint, E. W. Lindberg, M. O. Krucoff, J. M. Rosenow, and M. W. Slutzky, "Decoding human grasp kinematics and dynamics using epidural and subdural cortical signals," in Society for Neuroscience, Washington, DC, USA, 2011.

[111] W. Wang, J. L. Collinger, A. D. Degenhart et al., "An electrocorticographic brain interface in an individual with tetraplegia," PLoS ONE, vol. 8, no. 2, Article ID e55344, 2013.

[112] M. Hirata, T. Yanagisawa, K. Matsushita et al., "Brain-machine interface using brain surface electrodes: real-time robotic control and a fully implantable wireless system," in Technological Advancements in Biomedicine for Healthcare Applications, J. Wu, Ed., Okayama University, Okayama, Japan, 2012.

[113] H. Shibasaki and M. Kato, "Movement associated cortical potentials with unilateral and bilateral simultaneous hand movement," Journal of Neurology, vol. 208, no. 3, pp. 191-199, 1975.

[114] I. M. Tarkka and M. Hallett, "Cortical topography of premotor and motor potentials preceding self-paced, voluntary movement of dominant and non-dominant hands," Electroencephalography and Clinical Neurophysiology, vol. 75, no. 2, pp. 36-43, 1990.

[115] A. Urbano, C. Babiloni, P. Onorati et al., "Responses of human primary sensorimotor and supplementary motor areas to internally triggered unilateral and simultaneous bilateral one-digit movements: a high-resolution EEG study," European Journal of Neuroscience, vol. 10, no. 2, pp. 765-770, 1998.

[116] K. J. Wisneski, N. Anderson, G. Schalk, M. Smyth, D. Moran, and E. C. Leuthardt, "Unique cortical physiology associated with ipsilateral hand movements and neuroprosthetic implications," Stroke, vol. 39, no. 12, pp. 3351-3359, 2008.

[117] M. Sharma, C. Gaona, J. Roland, N. Anderson, Z. Freudenberg, and E. C. Leuthardt, "Ipsilateral directional encoding of joystick movements in human cortex," in Proceedings of the Annual International Conference of the IEEE Engineering in Medicine and Biology Society (EMBS '09), 2009.

[118] R. J. Seitz, P. Höflich, F. Binkofski, L. Tellmann, H. Herzog, and H.-J. Freund, "Role of the premotor cortex in recovery from middle cerebral artery infarction," Archives of Neurology, vol. 55, no. 8, pp. 1081-1088, 1998.

[119] C. Weiller, F. Chollet, K. J. Friston, R. J. S. Wise, and R. S. J. Frackowiak, "Functional reorganization of the brain in recovery from striatocapsular infarction in man," Annals of Neurology, vol. 31, no. 5, pp. 463-472, 1992.

[120] C. Bütefisch, H. Hummelsheim, P. Denzler, and K.-H. Mauritz, "Repetitive training of isolated movements improves the outcome of motor rehabilitation of the centrally paretic hand," Journal of the Neurological Sciences, vol. 130, no. 1, pp. 59-68, 1995.

[121] J. Farmer, X. Zhao, H. van Praag, K. Wodtke, F. H. Gage, and B. R. Christie, "Effects of voluntary exercise on synaptic plasticity 
and gene expression in the dentate gyrus of adult male spraguedawley rats in vivo," Neuroscience, vol. 124, no. 1, pp. 71-79, 2004.

[122] R. Colombo, F. Pisano, S. Micera et al., "Robotic techniques for upper limb evaluation and rehabilitation of stroke patients," IEEE Transactions on Neural Systems and Rehabilitation Engineering, vol. 13, no. 3, pp. 311-324, 2005.

[123] L. Dipietro, M. Ferraro, J. J. Palazzolo, H. I. Krebs, B. T. Volpe, and N. Hogan, "Customized interactive robotic treatment for stroke: EMG-triggered therapy," IEEE Transactions on Neural Systems and Rehabilitation Engineering, vol. 13, no. 3, pp. 325334, 2005.

[124] S. E. Fasoli, H. I. Krebs, J. Stein, W. R. Frontera, and N. Hogan, "Effects of robotic therapy on motor impairment and recovery in chronic stroke," Archives of Physical Medicine and Rehabilitation, vol. 84, no. 4, pp. 477-482, 2003.

[125] E. Swinnen, J. P. Baeyens, K. Knaepen et al., "Walking with robot assistance: the influence of body weight support on the trunk and pelvis kinematics," Disability and Rehabilitation: Assistive Technology, 2014.

[126] S. Masiero, P. Poli, G. Rosati et al., "The value of robotic systems in stroke rehabilitation," Expert Review of Medical Devices, vol. 11, pp. 187-198, 2014.

[127] H. I. Krebs and B. T. Volpe, "Rehabilitation robotics," in Handbook of Clinical Neurology, vol. 110 of Neurological Rehabilitation, chapter 23, pp. 283-294, Elsevier, 2013.

[128] H. I. Krebs, N. Hogan, M. L. Aisen, and B. T. Volpe, "Robotaided neurorehabilitation," IEEE Transactions on Rehabilitation Engineering, vol. 6, no. 1, pp. 75-87, 1998.

[129] B. T. Volpe, M. Ferraro, D. Lynch et al., "Robotics and other devices in the treatment of patients recovering from stroke," Current Atherosclerosis Reports, vol. 6, no. 4, pp. 314-319, 2004.

[130] X. L. Hu, K.-Y. Tong, R. Song, X. J. Zheng, and W. W. F. Leung, "A comparison between electromyography-driven robot and passive motion device on wrist rehabilitation for chronic stroke," Neurorehabilitation and Neural Repair, vol. 23, no. 8, pp. 837-846, 2009.

[131] F. Abdollahi, E. D. Case Lazarro, M. Listenberger et al., "Error augmentation enhancing arm recovery in individuals with chronic stroke: a randomized crossover design," Neurorehabilitation and Neural Repair, vol. 28, pp. 120-128, 2014.

[132] H. Kawamoto, S. Taal, H. Niniss et al., "Voluntary motion support control of Robot Suit HAL triggered by bioelectrical signal for hemiplegia," in Proceedings of the 32nd Annual International Conference of the IEEE Engineering in Medicine and Biology Society (EMBC '10), pp. 462-466, Buenos Aires, Argentina, September 2010.

[133] R. Colombo, I. Sterpi, A. Mazzone, C. Delconte, and F. Pisano, "Taking a lesson from patients' recovery strategies to optimize training during robot-aided rehabilitation," IEEE Transactions on Neural Systems and Rehabilitation Engineering, vol. 20, no. 3, pp. 276-285, 2012.

[134] A. Panarese, R. Colombo, I. Sterpi, F. Pisano, and S. Micera, "Tracking motor improvement at the subtask level during robot-aided neurorehabilitation of stroke patients," Neurorehabilitation and Neural Repair, vol. 26, no. 7, pp. 822-833, 2012.

[135] R. Song, K.-Y. Tong, X. Hu, and L. Li, "Assistive control system using continuous myoelectric signal in robot-aided arm training for patients after stroke," IEEE Transactions on Neural Systems and Rehabilitation Engineering, vol. 16, no. 4, pp. 371379, 2008.

[136] X. L. Hu, R. Song, K. Y. Tong, S. F. Tsang, P. O. Leung, and L. $\mathrm{Li}$, "Variation of muscle coactivation patterns in chronic stroke during robot-assisted elbow training," Archives of Physical Medicine and Rehabilitation, vol. 88, no. 8, pp. 1022-1029, 2007.

[137] X. L. Hu, K. Y. Tong, R. Song et al., "Quantitative evaluation of motor functional recovery process in chronic stroke patients during robot-assisted wrist training," Journal of Electromyography \& Kinesiology, vol. 19, no. 4, pp. 639-650, 2009.

[138] X. L. Hu, K. Y. Tong, R. Li, J. J. Xue, S. K. Ho, and P. N. Chen, "Combined functinal electrical stimulation (FES) and robotic system driven by user intention for post-stroke wrist rehabilitation," in Biomechantronics in Medicine and Health Care, K. Y. Tong, Ed., Pan Stanford Publishing, Singapore, 2011.

[139] "Home health care services-industry report," NAICS, vol. 621610, 2012.

[140] A. Gonzalez, I. Nambu, H. Hokari, and Y. Wada, "EEG channel selection using particle swarm optimization for the classification of auditory event-related potentials," The Scientific World Journal, vol. 2014, Article ID 350270, 11 pages, 2014.

[141] W.-K. Tam, K.-Y. Tong, F. Meng, and S. Gao, "A minimal set of electrodes for motor imagery BCI to control an assistive device in chronic stroke subjects: a multi-session study," IEEE Transactions on Neural Systems and Rehabilitation Engineering, vol. 19, no. 6, pp. 617-627, 2011.

[142] B. Lanfer, C. Röer, M. Scherg, S. Rampp, C. Kellinghaus, and C. Wolters, "Influence of a silastic ECoG grid on EEG/ECoG based source analysis," Brain Topography, vol. 26, no. 2, pp. 212-228, 2013.

[143] A. Zimmermann-Schlatter, C. Schuster, M. A. Puhan, E. Siekierka, and J. Steurer, "Efficacy of motor imagery in poststroke rehabilitation: a systematic review," Journal of NeuroEngineering and Rehabilitation, vol. 5, article 8, 2008.

[144] R. S. Menon and S.-G. Kim, "Spatial and temporal limits in cognitive neuroimaging with fMRI," Trends in Cognitive Sciences, vol. 3, no. 6, pp. 207-216, 1999.

[145] T. C. Ferree, M. T. Clay, and D. M. Tucker, "The spatial resolution of scalp EEG," Neurocomputing, vol. 38-40, pp. 1209-1216, 2001.

[146] E. Asano, C. Juhász, A. Shah et al., "Origin and propagation of epileptic spasms delineated on electrocorticography," Epilepsia, vol. 46, no. 7, pp. 1086-1097, 2005. 


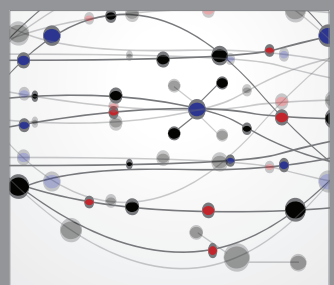

The Scientific World Journal
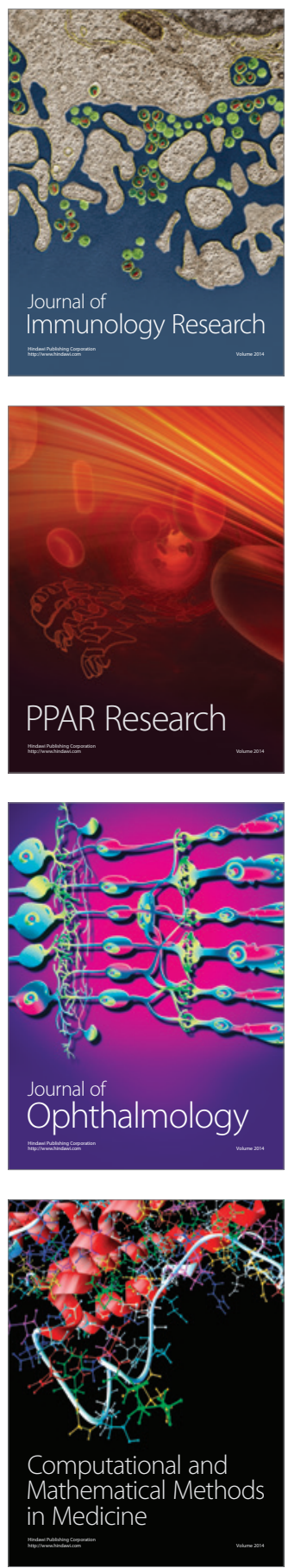

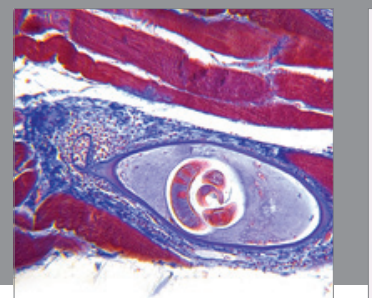

Gastroenterology

Research and Practice
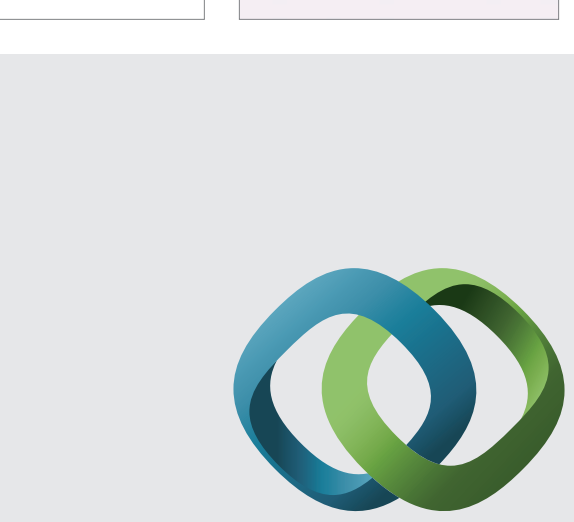

\section{Hindawi}

Submit your manuscripts at

http://www.hindawi.com
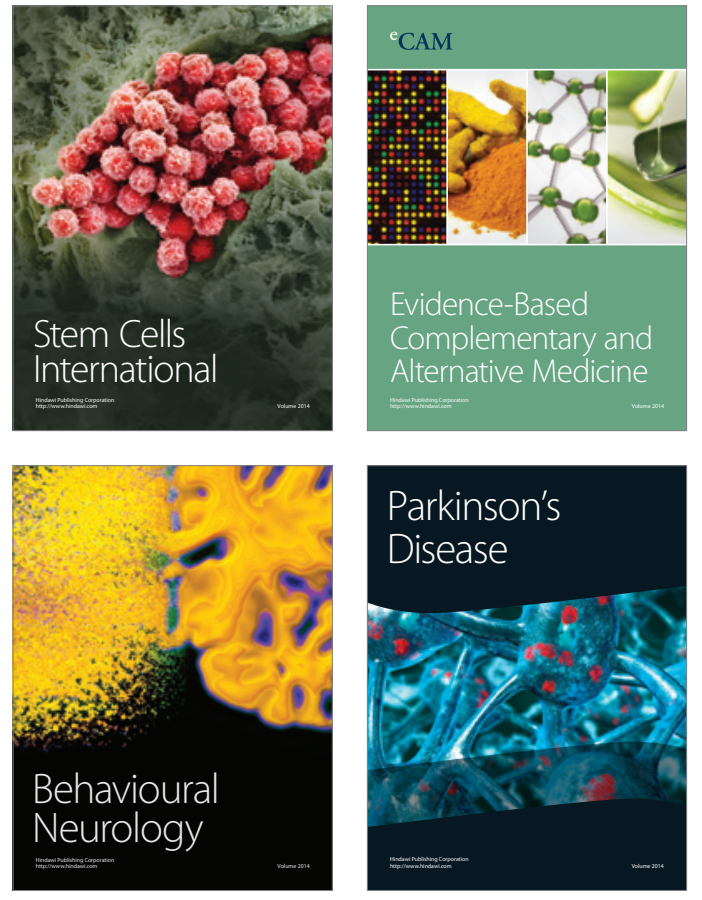
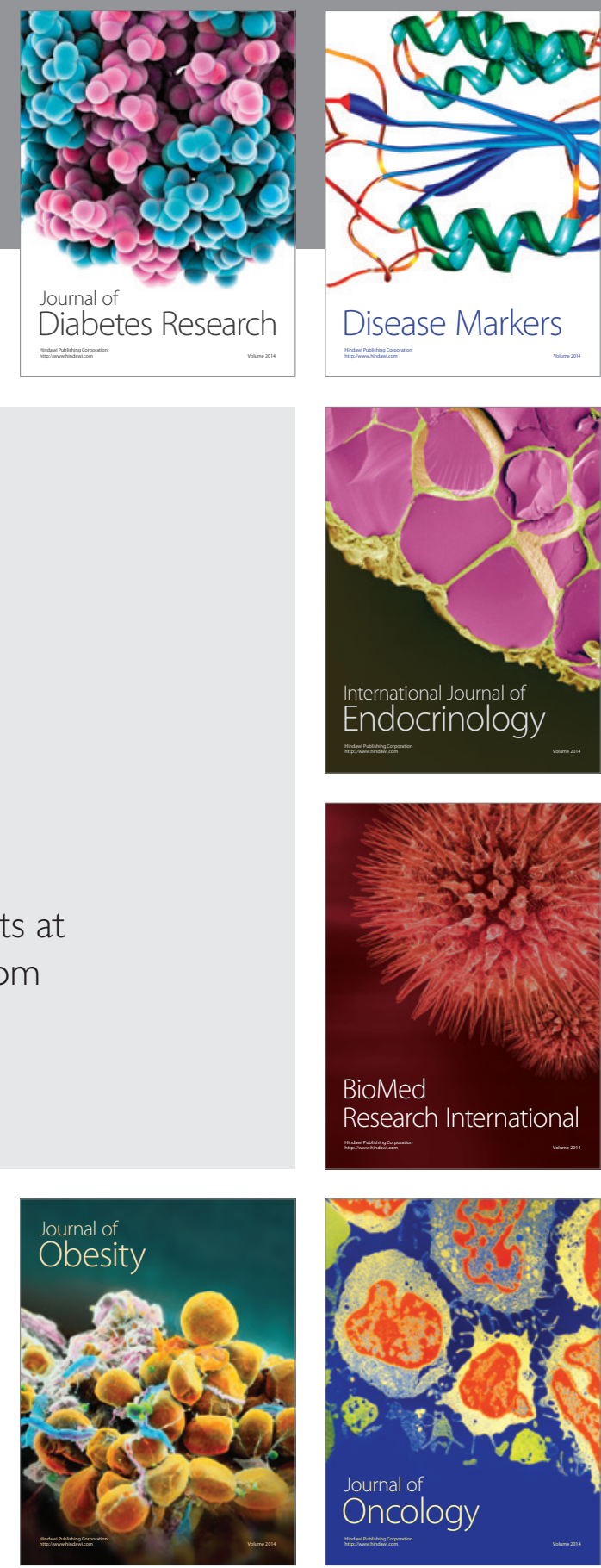

Disease Markers
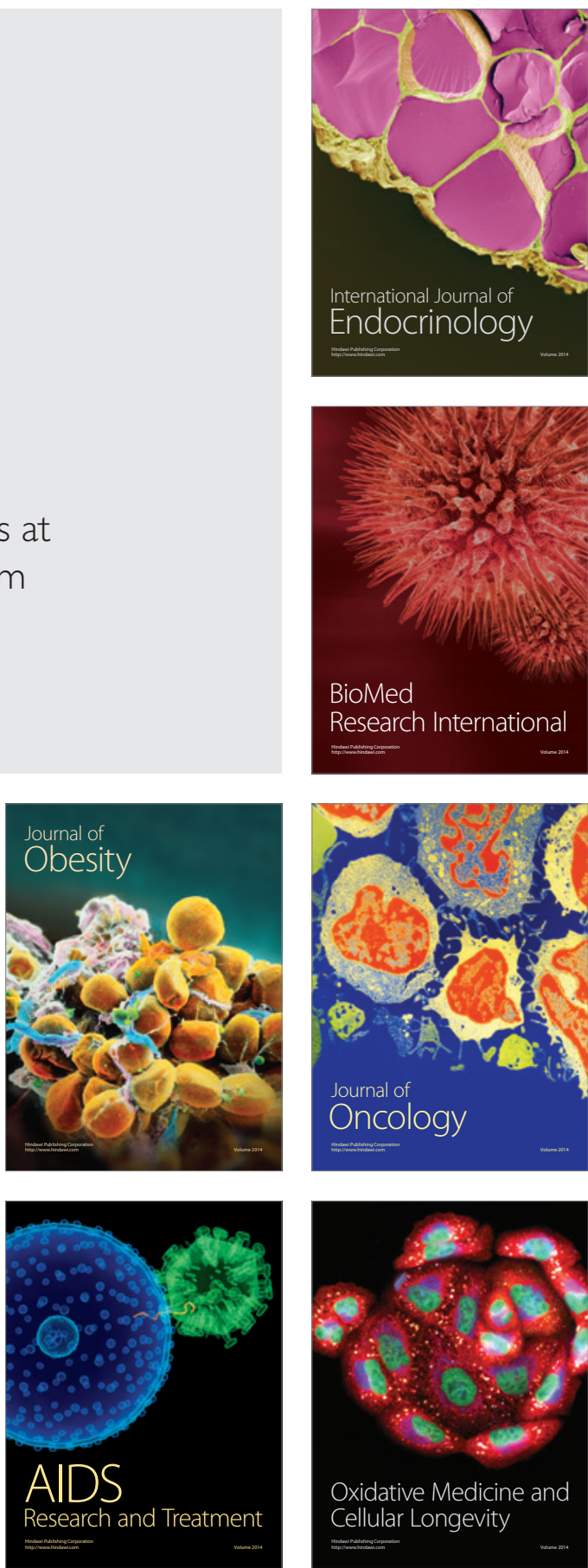